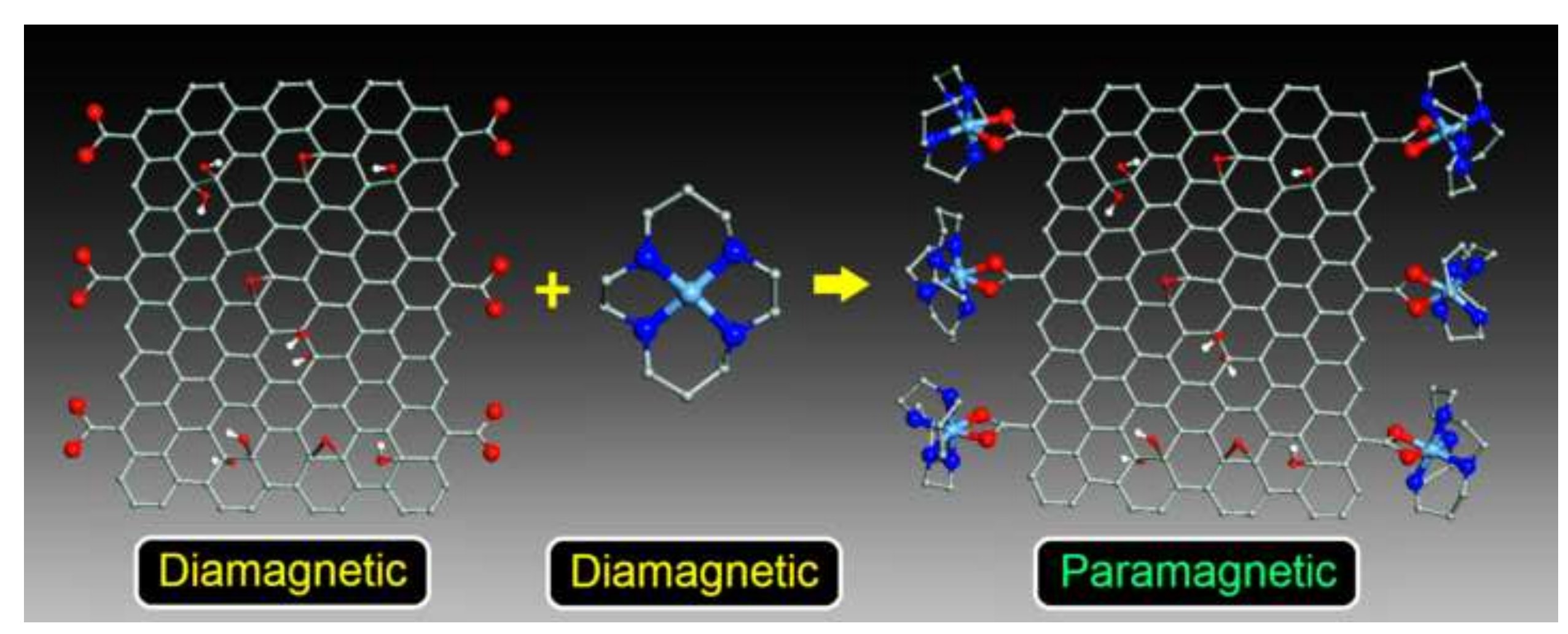

Diamagnetic
Diamagnetic

$+\hat{x} \rightarrow$

Paramagnetic

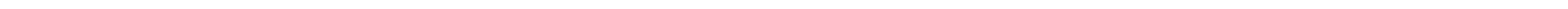




\section{Coordination functionalization of graphene oxide with tetraazamacrocyclic complexes of nickel(II): Generation of paramagnetic centers ${ }^{\dagger}$}

Vladimir A. Basiuk, ${ }^{\mathrm{a}, \mathrm{b}}$ Natalia Alzate-Carvajal, ${ }^{\mathrm{c}}$ Laura V. Henao-Holguín, ${ }^{\mathrm{a}}$ Elena V. RybakAkimova $^{\mathrm{b}}$ and Elena V. Basiuk ${ }^{\mathrm{b}, \mathrm{c}}$

${ }^{a}$ Instituto de Ciencias Nucleares, Universidad Nacional Autónoma de México, Circuito Exterior C.U., 04510 México D.F., Mexico

${ }^{\mathrm{b}}$ Department of Chemistry,Tufts University, 62 Talbot Avenue, Medford, MA 02155, USA

${ }^{\mathrm{c}}$ Centro de Ciencias Aplicadas y Desarrollo Tecnológico, Universidad Nacional Autónoma de México, Circuito Exterior C.U., 04510 México D.F., Mexico

* Address correspondence to: elbg1111@gmail.com, basiuk@ nucleares.unam.mx

${ }^{\dagger}$ Electronic supplementary information available: Comparison of FTIR spectra for GO before and after treatment with $\mathrm{NH}_{4} \mathrm{OH}$ solution of $\mathrm{pH} 12$ without $\mathrm{Ni}(\mathrm{II})$ tetraazamacrocyclic complexes (control experiment); Raman and EPR spectra for $\mathrm{GO}, \mathrm{GO}+[\mathrm{Ni}($ cyclam)] and $\mathrm{GO}+[\mathrm{Ni}($ tet $b)]$; XPS spectra (survey and deconvolution for C 1s and O 1s) of GO before functionalization; the data of magnetic susceptibility measurements; total energies calculated by DFT for GO model, macrocyclic dications $[\mathrm{Ni}(\text { cyclam })]^{2+}$ and $[\mathrm{Ni}(\text { tet } b)]^{2+}$, and the high-spin complexes $\mathrm{GO}+[\mathrm{Ni}($ cyclam $)]$ and $\mathrm{GO}+[\mathrm{Ni}($ tet $b)]$ 


\begin{abstract}
We describe a novel approach to functionalization of graphene oxide (GO) which allows for a facile generation of paramagnetic centers from two diamagnetic components. Coordination attachment of $[\mathrm{Ni}(\text { cyclam })]^{2+}$ or $[\mathrm{Ni}(\text { tet } b)]^{2+}$ tetraazamacrocyclic cations to carboxylic groups of GO takes place under basic conditions in aqueous-based reaction medium. The procedure is very straightforward and does not require high temperatures or other harsh conditions. Changing the coordination geometry of $\mathrm{Ni}$ (II) from square-planar tetracoordinated to pseudooctahedral hexacoordinated brings about the conversion from low-spin to high-spin state of the metal centers. Even though the content of tetraazamacrocyclic complexes in functionalized GO samples was found to be relatively low (nickel content of ca. $1 \mathrm{wt} \%$, as determined by thermogravimetric analysis, elemental analysis and energy dispersive X-ray spectroscopy), room temperature magnetic susceptibility measurements easily detected the appearance of paramagnetic properties in $\mathrm{GO}+[\mathrm{Ni}($ cyclam) $]$ and $\mathrm{GO}+[\mathrm{Ni}(\operatorname{tet} b)]$ nanohybrids, with effective magnetic moments of 1.95 BM and 2.2 BM for, respectively. According to density functional theory calculations, the main spin density is localized at the macrocyclic complexes, without considerable extension to graphene sheet, which suggests insignificant ferromagnetic coupling in the nanohybrids, in agreement with the results of magnetic susceptibility measurements. The coordination attachment of $\mathrm{Ni}(\mathrm{II})$ tetraazamacrocycles to $\mathrm{GO}$ results in considerable changes in Fourier-transform infrared and X-ray photoelectron spectra, as well as in GO morphology, as observed by scanning and transmission electron microscopy, atomic force and scanning tunneling microscopy.
\end{abstract}

Keywords: Graphene oxide; Coordination functionalization; Tetraazamacrocyclic complexes; Nickel(II); High-spin; Density functional theory 


\section{Introduction}

Tuning electronic and magnetic properties of graphene-based materials by chemical functionalization is a subject of increasing research effort, with particular emphasis on the design of new efficient heterogeneous catalysts, solar energy conversion systems, sensors, contrast agents for biomedical imaging, and photoluminescent, nanoelectronic and spintronic devices [1-7]. Among the most important classes of such nanohybrids are those incorporating azamacrocyclic ligands and their complexes (primarily porphyrins and phthalocyanins), where electronic structure, spectroscopic or redox properties, and reactivity can be easily modulated by variations of the metal centers; cyclic structure of the ligands prevents potential leakage of the encapsulated metal. In order to attach these molecules to graphene and graphene oxide (GO), different types of interactions can be exploited, usually covalent (for example, amide) bonding, noncovalent ( $\pi-\pi$ stacking and van der Waals) or electrostatic interactions [1,2,4-6,8-16]. However, covalent functionalization alters electronic structure of carbon support, while relatively weak non-covalent interactions may lead to easy removal of deposited molecules (especially upon treating with solvents). In addition to the covalent functionalization through amide formation between $\mathrm{COOH}$ groups of $\mathrm{GO}$ and amino-substituted azamacrocycles $[1,2,4,10]$, the rich chemistry of oxidized graphene offers the possibility of complexation between its oxygen-containing (i.e., carboxylic) functionalities and coordinatively unsaturated central metal atoms of macrocyclic complexes. In a recent report by Jurow et al. [17], the protruding oxophilic central metal ion of $\mathrm{Zr}(\mathrm{IV})$ porphyrinoids was axially coordinated to the oxygen bearing functional groups on GO surfaces to result in new supramolecular photonic materials with a high degree of macrocycle attachment onto graphene sheet edges and large defects. Wang et al. [18] prepared nanohybrids with enhanced nonlinear optical properties by anchoring a $\mathrm{Sn}(\mathrm{IV})$ porphyrin bearing an $\mathrm{OH}$ axial ligand onto thionyl chloride-activated GO. Axial 
coordination as a convenient, mild method of graphene functionalization deserves further exploration.

The fabrication of nanohybrids of graphene and GO with tetraazamacrocyclic complexes exhibiting magnetic properties is a topic of special interest. It is related mainly to organic spin electronic (spintronic) devices, where a bit of information is represented by a single magnetic moment of molecules or atoms deposited on graphene surface. Single magnetic atoms on surfaces, albeit showing enhanced magnetic anisotropy which stabilizes the magnetic structure, tend to aggregate (coalesce) on surfaces without forming regular patterns and can present a decreased magnetic response due to the interaction with the surface (see [19] and references therein). On the contrary, the deposition of magnetic metal atoms in the form of complexes with organic ligands constitute an efficient approach to achieving regular and stable networks of magnetic units. Different combinations of organic ligands and paramagnetic central metal ions proved to be useful to fabricate such magnetic nanohybrids. The complexes with relatively simple bidentate and tridentate ligands can be exemplified by $\left[\mathrm{Fe}(\mathrm{bpz})_{2}\right.$ phen $](\mathrm{bpz}=$ dihydrobis (pyrazolyl)borate; phen $=1,10$ phenanthroline) deposited onto highly oriented pyrolytic graphite, which exhibited highly efficient thermal and light-induced spin-state switching [20], as well as complexes [Fe(qsal $\left.)_{2}\right]^{+}$and $\left[\mathrm{Fe}(\mathrm{qnal})_{2}\right]^{+}\left(\mathrm{qsal}^{-}=(N-(8-\text { quinolyl })-\text { salicylaldimine })^{-} ;\right.$qnal $=(1-((8-$ quinolinylimino $)$ methyl $)-2-$ naphthalenol) $)^{-}$) forming ionic spin crossover nanohybrids with the negatively charged GO [21]. However, the main interest is directed towards paramagnetic metal complexes with tetraazamacrocyclic porphyrin [12,22-24] and phthalocyanine [19,25-27] ligands. In addition to their exceptional stability, such kind of molecular doping allows for tuning the electrical properties of the graphene sheet, on one hand, while on the other hand, graphene can drive molecular self-assembly of macrocyclic units, and serve as a tool to switch their molecular spin state [26]. Since the interaction is mainly of van der Waals nature, graphene acts as a decoupling layer to efficiently 
preserve the electrical and structural properties of the tetraazamacrocyclic complexes [19,22-26]. The commonly studied molecules incorporate $3 d$ metal atoms such as iron(II) $[19,23,25,26]$ and cobalt(II) [22,24]. Candini et al. [27] reported a different example, with a $4 f$ element, and designed a novel hybrid spintronic nanodevice made by noncovalent decoration with a terbium(III) bis(phthalocyanine) complex, to electrically detect the magnetization reversal of the molecules in proximity with graphene.

Spintronics is not the only possible application area for graphene and GO-supported magnetic tetraazamacrocyclic complexes, likewise porphyrins and phthalocyanines are not the only macrocyclic ligands of interest. Recently, gadolinium(III) complexes with a series of 15 substituted saturated tetraazamacrocyclic ligands were tested for noncovalent functionalization of GO [28]. The magnetic nanohybrids obtained in this way enhanced the contrast properties of Gd(III) for cellular magnetic resonance imaging.

In all the related studies performed until now, the functionalizing tetraazamacrocyclic complexes were employed which already possess magnetic properties due to the presence of paramagnetic central metal ions. At the same time, a possibility exists to in situ generate magnetic properties in GO by using diamagnetic complexes of common transition metal ions such as nickel(II). Among polyazamacrocyclic ligands known for about half a century, there is a family of saturated 14-membered tetraazamacrocycles, including 1,4,8,11-tetraazacyclotetradecane and rac5,5,7,12,12,14-hexamethyl-1,4,8,11-tetraazacyclotetradecane (see review [29] and references therein), commonly referred to as cyclam and tet $b$, respectively. Their complexes with some transition metals, particularly with $\mathrm{Ni}(\mathrm{II})$, exhibit interesting and potentially useful properties associated with conformational flexibility of the saturated macrocyclic ring, which cannot be found in rigid hyperconjugated porphyrin and phthalocyanine molecules. Specifically, low-spin squareplanar cations $[\mathrm{Ni}(\mathrm{cyclam})]^{2+}$ and $[\mathrm{Ni}(\text { tet } b)]^{2+}$ in basic media can additionally coordinate 
carboxylate ions as bidentate ligands, thus converting into high-spin pseudooctahedral complexes (Scheme 1) [29-32].

Due to the abundance of $\mathrm{COOH}$ groups on sheet edges $[1,2,4]$, GO can be considered as a carboxylic ligand capable of coordinating to $[\mathrm{Ni}(\text { cyclam })]^{2+}$ and $[\mathrm{Ni}(\text { tet } b)]^{2+}$ tetraazamacrocyclic cations. The resulting complexation reaction can be envisioned as a very simple way of generating paramagnetic centers in GO by treating it with diamagnetic reagents. The present study was undertaken to verify the feasibility of the above approach. In particular, we prepared two nanohybrids, hereafter referred to as $\mathrm{GO}+[\mathrm{Ni}($ cyclam $)]$ and $\mathrm{GO}+[\mathrm{Ni}($ tet $b)]$, and performed their detailed characterization by a variety of analytical techniques, including Fourier-transform infrared (FTIR), Raman, electron paramagnetic resonance (EPR), energy dispersive X-ray (EDS) and X-ray photoelectron spectroscopy (XPS), thermogravimetric (TGA) and elemental analysis, magnetic susceptibility measurements, scanning and transmission electron microscopy (SEM and TEM, respectively), atomic force and scanning tunneling microscopy (AFM and STM, respectively). Density functional theory (DFT) calculations were employed to provide an additional insight into the structure and properties of $\mathrm{GO}+[\mathrm{Ni}($ cyclam $)]$ and $\mathrm{GO}+[\mathrm{Ni}($ tet $b)]$ nanohybrids.

\section{Experimental and theoretical methods}

\subsection{Materials}

We used GO nanopowder from Nanostructured and Amorphous Materials, Inc.; purity >99 wt $\%$, sheet size 0.5-3 $\mu \mathrm{m}, 1-10$ layers of 0.55-1.2 nm thickness. Cyclam $(1,4,8,11-$ tetraazacyclotetradecane; purity $98 \%), \mathrm{Ni}(\mathrm{II})$ acetate tetrahydrate $\left(\mathrm{Ni}\left(\mathrm{OCOCH}_{3}\right)_{2} \cdot 4 \mathrm{H}_{2} \mathrm{O}, 98 \%\right)$, perchloric acid $\left(\mathrm{HClO}_{4}, 70 \%\right)$, ammonium hydroxide solution $\left(\mathrm{NH}_{4} \mathrm{OH}, 28-30 \%\right)$, methanol (anhydrous $\mathrm{CH}_{3} \mathrm{OH}, 99.8 \%$ ), ethanol (anhydrous $\mathrm{C}_{2} \mathrm{H}_{5} \mathrm{OH}, \geq 99.8 \%$ ) and isopropanol (anhydrous $\mathrm{C}_{3} \mathrm{H}_{7} \mathrm{OH}, 99.5 \%$ ) were purchased from Sigma-Aldrich, Inc., and used as received. Free ligand tet $b$ 
was prepared according to the procedure described elsewhere [33,34]. Tetraazamacrocyclic complexes $[\mathrm{Ni}($ cyclam $)]\left(\mathrm{ClO}_{4}\right)_{2}$ and $[\mathrm{Ni}($ tet $b)]\left(\mathrm{ClO}_{4}\right)_{2}$ were synthesized by complexation of the free ligands with $\mathrm{Ni}(\mathrm{II})$ acetate tetrahydrate in methanol followed by addition of perchloric acid [34,35].

\subsection{Functionalization of $G O$}

$\mathrm{Ni}$ (II) tetraazamacrocyclic complex perchlorate $(20 \mathrm{mg})$ was dissolved in water $(6 \mathrm{~mL})$ producing solution of yellow color typical for the nickel(II) complexes with square-planar coordination. Separately, GO (200 mg) was dispersed in ethanol (4 mL), and the solution of macrocyclic complex was added to the $\mathrm{GO}$ suspension. The $\mathrm{pH}$ was adjusted to ca. 12 with $\mathrm{NH}_{4} \mathrm{OH}$. The resulting suspension was heated at $70{ }^{\circ} \mathrm{C}$ on a heating plate for $5 \mathrm{~h}$, then left overnight at ambient temperature. The $\mathrm{pH}$ was adjusted again to ca. 12 with $\mathrm{NH}_{4} \mathrm{OH}$. Then the solid phase (functionalized GO) was filtered out, washed with ethanol and dried in a vacuum desiccator.

\subsection{Characterization}

FTIR spectra were acquired using a Nexus 670 FTIR Thermo-Nicolet instrument, equipped with an Olympus BX52 microscope, under room temperature and atmospheric pressure. Raman spectra were recorded on a Thermo-Nicolet Almega Dispersive Raman instrument $(\Lambda=532 \mathrm{~nm})$. For XPS studies, we employed a Thermo Scientific ${ }^{\mathrm{TM}}$ ESCALAB $^{\mathrm{TM}}$ 250Xi X-ray Photoelectron Spectrometer Microprobe, equipped with a monochromated Al K $\alpha$ X-ray source with energy of $1486.6 \mathrm{eV}$; work function of the spectrometer, $4.2 \mathrm{eV}$. EPR spectra were recorded using a Bruker EMX X-band spectrometer, at $9.319 \mathrm{GHz}$ frequency. TGA curves were acquired by using a SDTQ600 analyzer from TA Instruments, under air flow of $100 \mathrm{ml} \mathrm{min}^{-1}$ and with a heating ramp of 10

${ }^{\circ} \mathrm{C} \min ^{-1}$. SEM characterization was carried out on a JEOL JSM-6510LV instrument operating at 20 $\mathrm{kV}$, coupled to an EDS INCA Energy 250 Energy Dispersive X-ray Microanalysis System from 
Oxford Instruments. For TEM observations, a JEOL 4000EX microscope was employed, operating at $200 \mathrm{kV}$. AFM images were acquired using a JEOL JSPM-5200 microscope, in tapping mode on samples deposited onto mica. STM studies were done on the same instrument on samples deposited onto highly-oriented pyrolytic graphite (HOPG supports from Nanoscience Instruments, Inc.), in the constant current mode at room temperature using mechanically cut $\mathrm{Pt} / \mathrm{Ir}$ (diameter of $0.25 \mathrm{~mm}$ ) tips.

Magnetic susceptibility of the powdered samples was measured at room temperature using portable Evans magnetic susceptibility balance (Johnson Matthey). Each sample was gently packed in the pre-weighted sample tube provided with the instrument, then the length of the sample layer in the sample tube, $L$, was measured (in $\mathrm{cm}$ ), and the tube with the sample was weighted again. After zeroing the balance, the tube with the sample was inserted into the sample holder, and the reading of the digital display $(R)$ was recorded. The reading for the empty tube, $R_{0}$, was recorded prior to manipulations with the sample. Mass susceptibility of each sample was calculated using the equation $\chi_{g}=\frac{C L\left(R-R_{o}\right)}{m \times 10^{9}}$. Molar susceptibility per nickel was calculated from experimentally determined mass susceptibility and the results of elemental microanalysis for nickel:

$\chi_{m}=\chi_{g} \times 100 \times M(N i) / \% N i \quad($ where $\mathrm{M}(\mathrm{Ni})=58 \mathrm{~g} / \mathrm{mol})$

Effective magnetic moment (per nickel) was calculated using the following formula: $\mu_{\text {eff }}=2.828 \sqrt{\chi_{m} T}$

\subsection{Theoretical calculations}

The DFT calculations were performed by using the DMol3 numerical-based densityfunctional module implemented in the Materials Studio 6.0 software package from Accelrys Inc. [36-39]. The computational methodology of choice was a general gradient approximation (GGA) functional PBE (Perdew-Burke-Ernzerhof correlation [40]) with Grimme dispersion correction [41] 
in conjunction with the double numerical basis set DNP [39], which has a polarization $d$-function added on all non- $\mathrm{H}$ atoms and a polarization $p$-function added on all $\mathrm{H}$ atoms. This combination was shown to be an efficient calculation technique in the frame of DMol3 module for the description of noncovalent interactions between tetraazamacrocyclic compounds with carbon nanoclusters (fullerenes, fulleroids and oxidized graphene sheets) [42-44]. All-electron core treatment, a global orbital cutoff of $4.5 \AA$ and Fermi orbital occupancy (no thermal smearing) were applied. Full geometry optimization and calculation of electronic parameters was carried out by using the quality and convergence criteria set to 'fine'.

The formation energies $\Delta E_{\mathrm{GO}+\mathrm{Ni}}$ for $\mathrm{GO}+[\mathrm{Ni}($ cyclam $)]$ and $\mathrm{GO}+[\mathrm{Ni}($ tet $b)]$ complexes were calculated according to the following formula:

$\Delta E_{\mathrm{GO}+\mathrm{Ni}}=E_{\mathrm{GO}+\mathrm{Ni}}-\left(E_{\mathrm{Ni}}+E_{\mathrm{GO}}\right)$

where $E_{\mathrm{GO}}$ is the corresponding absolute energy for $\mathrm{GO}$, and $E_{\mathrm{Ni}}$, for $[\mathrm{Ni}(\operatorname{cyclam})]^{2+}$ or $[\mathrm{Ni}(\text { tet } b)]^{2+}$.

\section{Results and discussion}

Coordination functionalization of $\mathrm{GO}$ with $\mathrm{Ni}(\mathrm{II})$ tetraazamacrocyclic cations via the formation of high-spin octahedral carboxylates with $\mathrm{COOH}$ groups of oxidized graphene sheet edges was performed at pH 12 (ammonia solution), as illustrated in Scheme 2. Starting $[\mathrm{Ni}(\text { cyclam })]^{2+}$ and $[\mathrm{Ni}(\text { tet } b)]^{2+}$ complexes were employed as diperchlorate salts: half of the anions were substituted by carboxylates of $\mathrm{GO}$, and another half, by $\mathrm{OH}^{-}$anions present in a large excess in the ammonia basic medium.

A comparison of solubility/dispersibility behavior of nanomaterials before and after functionalization is the simplest test for changes in their chemical structure (an improved solubility of graphene-based materials due to chemical modification is a commonly known and sought-after effect $[1,2,5,15])$. We performed the test in isopropanol and water. As shown in Figure 1, GO is well 
dispersed in both solvents, giving brown solutions, the aqueous one being more stable without showing any visible changes after $24 \mathrm{~h}$. On the contrary, $\mathrm{GO}+[\mathrm{Ni}($ cyclam $)]$ and $\mathrm{GO}+[\mathrm{Ni}($ tet $b)]$ samples become totally insoluble in isopropanol, which can be attributed to the introduction of a large number of ions into GO structure. The latter factor can hardly affect the solubility behavior in aqueous solutions. Nevertheless, the dispersions of functionalized samples in water also turned to be less stable than that of starting GO, which can be explained by an increased interaction between functionalized graphene sheets (through electrostatic attraction of oppositely charged ionic species, e.g., between macrocyclic cation attached to one sheet and carboxylate anions of adjacent sheet), as well as by a rather hydrophobic nature of cyclam and tet $b$ ligands.

Dramatic changes due to the functionalization were observed in FTIR spectra (Figure 2). Apart from a very broad hydroxyl $v_{\mathrm{OH}}$ absorption around $3400 \mathrm{~cm}^{-1}$ and the corresponding $\delta_{\mathrm{OH}}$ band at $1628 \mathrm{~cm}^{-1}$, the most important spectral feature of GO is the $v_{\mathrm{C}=\mathrm{O}}$ band at $1736 \mathrm{~cm}^{-1}$ due to hydrogen-bonded $\mathrm{COOH}$ groups. The coordination attachment of $[\mathrm{Ni}(\mathrm{cyclam})]^{2+}$ or $[\mathrm{Ni}(\mathrm{tet} b)]^{2+}$ cations gives rise to a total disappearance of the latter band, instead of which a very intense absorption due to carboxylate ions $\left(v^{\mathrm{as}} \mathrm{COO}^{-}\right)$can be seen with a maximum at $1597 \mathrm{~cm}^{-1}$ for $\mathrm{GO}+[\mathrm{Ni}($ cyclam $)]$ and at $1618 \mathrm{~cm}^{-1}$ for $\mathrm{GO}+[\mathrm{Ni}($ tet $b)]$. The carboxylate absorption $v^{\mathrm{as}} \mathrm{COO}^{-}$after treatment of $\mathrm{GO}$ with $\mathrm{NH}_{4} \mathrm{OH}$ solution of $\mathrm{pH} 12$ without $\mathrm{Ni}(\mathrm{II})$ tetraazamacrocyclic complexes (control experiment; Figure S1 of Supporting Information) is observed as well, however the intensity of the corresponding band at $1620 \mathrm{~cm}^{-1}$ is much lower. In addition, the $v_{\mathrm{C}=\mathrm{O}}$ band due to hydrogen-bonded $\mathrm{COOH}$ groups (at $c a .1740 \mathrm{~cm}^{-1}$; Figure S1) did not disappear completely, in contrast to the spectra of $\mathrm{GO}+[\mathrm{Ni}($ cyclam $)]$ and $\mathrm{GO}+[\mathrm{Ni}($ tet $b)]$ (Figure 2$)$. The $v^{\mathrm{s}} \mathrm{COO}^{-}$vibrations are usually observed at ca. $1430 \mathrm{~cm}^{-1}$, where they apparently overlap with bending modes of $\mathrm{CH}_{2}$ and $\mathrm{CH}_{3}$ groups in the tetraazamacrocyclic ligands (Figure 2). For comparison, in the FTIR spectrum of bridged high-spin complex bis-[Ni(II)(tet $b)]$-2,5-pyridinedicarboxylate diperchlorate monohydrate, 
the $v^{\text {as }}{ }_{\mathrm{COO}^{-}}$and $v^{\mathrm{s}} \mathrm{COO}^{-}$vibrations were observed at 1609 and $1458 \mathrm{~cm}^{-1}$, respectively. The $\mathrm{CH}_{2}$ and $\mathrm{CH}_{3}$ groups in cyclam and tet $b$ ligands additionally manifest themselves as aliphatic $v^{\mathrm{s}}{ }_{\mathrm{CH}}$ and $v^{\mathrm{as}}{ }_{\mathrm{CH}}$ bands at about 2850 and $2920 \mathrm{~cm}^{-1}$, respectively [31]. Broad but intense bands around $3300 \mathrm{~cm}^{-1}$ reflect the presence of a large number of $\mathrm{N}-\mathrm{H}$ bonds $\left(v_{\mathrm{NH}}\right)$ in nanohybrids: most of them belong to cyclam and tet $b$ ligands (absorption centered at ca. 3340 and $3380 \mathrm{~cm}^{-1}$, respectively), with a notable contribution of $\mathrm{NH}_{4}^{+}$cations (at $3250-3260 \mathrm{~cm}^{-1}$ ) forming salts with residual $\mathrm{COO}^{-}$, which did not coordinate to tetraazamacrocyclic complexes. In the control experiment (GO treatment with $\mathrm{NH}_{4} \mathrm{OH}$ solution without $\mathrm{Ni}(\mathrm{II})$ tetraazamacrocyclic complexes; Figure $\mathrm{S} 1$ ), the latter band is observed at about $3220 \mathrm{~cm}^{-1}$, with no evident absorption at $3340-3380 \mathrm{~cm}^{-1}$. The bands at $1050-1070$ $\mathrm{cm}^{-1}$ are due to $v_{\mathrm{C}-\mathrm{O}}$ in epoxide and alcohol moieties of GO.

Raman spectra of the functionalized GO samples are generally featureless, showing no new bands due to the tetraazamacrocyclic cations (Figure S2 of Supporting Information). All the spectra exhibit disorder (D) and graphite (G) bands at about 1355 and $1590 \mathrm{~cm}^{-1}$, respectively, typical for GO $[11,13,15,45]$ as well as for carbon nanotubes containing a large number of structural defects with carbon atoms in $s p^{3}$ hybridization state. The $2 \mathrm{D}$ and $\mathrm{D}+\mathrm{G}$ overtone bands [11] clearly seen in the spectrum of GO at 2683 and $2910 \mathrm{~cm}^{-1}$, respectively, become poorly distinguishable for the functionalized samples.

The most detailed spectral information on chemical bonds in the functionalized GO samples was afforded by XPS technique (for comparison, Figure S3 of Supporting Information shows XPS spectrum of GO before functionalization). As it was expected, the spectra of both nanohybrids (Figure 3 ) show the lines due to $\mathrm{C} 1 \mathrm{~s}, \mathrm{~N} 1 \mathrm{~s}, \mathrm{O} 1 \mathrm{~s}$ and Ni $2 \mathrm{p}$ (split into Ni $2 \mathrm{p}_{1 / 2}$ and Ni $2 \mathrm{p}_{3 / 2}$ components). On the other hand, no $\mathrm{Cl} 2 \mathrm{p}$ signature was detected in the range of 196-204 eV, which implies that the perchlorate anions were indeed completely substituted by the coordinated $\mathrm{COO}^{-}$ groups of $\mathrm{GO}$ and $\mathrm{OH}^{-}$anions of the ammonia basic medium. Deconvolution analysis of the $\mathrm{C} 1 \mathrm{~s}$ 
peak reveals the presence of three main types of carbon atoms characteristic for GO, which are $s p^{2}$ and $s p^{3} \mathrm{C}-\mathrm{C}$ carbons (binding energy of $284.7 \mathrm{eV}$ ), epoxide $\mathrm{C}-\mathrm{O}-\mathrm{C}(286.6 \mathrm{eV})$, and carbonyl $\mathrm{C}=\mathrm{O}$, including those found in carboxylates $(287.7-287.9 \mathrm{eV})[2,4,11,13,15,45]$. Aliphatic carbon atoms $\left(s p^{3}\right)$ including those belonging to the macrocycles cannot be reliably separated, however the intensity of $\mathrm{C}-\mathrm{C}$ peak increased considerably for the nanohybrids as compared to the spectrum of GO before functionalization (Figure S3). Deconvolution of the $\mathrm{N}$ 1s peak for both samples gives two components with a binding energy of 398.7 and $400.1 \mathrm{eV}$, which are typical for polyazamacrocyclic compounds, including porphyrins and phthalocyanines [46-50]. In the case of O $1 \mathrm{~s}$, the components characteristic for $\mathrm{GO}$ are due to oxygen atoms in $\mathrm{C}=\mathrm{O}$ and $\mathrm{O}-\mathrm{C}=\mathrm{O}$ groups (binding energy of $532.4 \mathrm{eV}$ ), in alcohol $\mathrm{C}-\mathrm{O}-\mathrm{H}$ and epoxide $\mathrm{C}-\mathrm{O}-\mathrm{C}$ groups (533.1-533.2 eV), and in carboxylic functionalities (533.5-533.6 eV) [4]. In addition, a prominent band at $531.3 \mathrm{eV}$ was found (absent in the spectrum of GO before functionalization; Figure S3), which was attributed to $\mathrm{Ni}-\mathrm{O}$ bonds [51-53] (particularly with carboxylate anions [52,53]), and thus is very important to confirm the successful complexation between $\mathrm{COOH}$ groups of $\mathrm{GO}$ and tetraazamacrocyclic cations $[\mathrm{Ni}(\text { cyclam })]^{2+}$ and $[\mathrm{Ni}(\text { tet } b)]^{2+}$. Finally, deconvolution of the $\mathrm{Ni} 2 \mathrm{p}_{3 / 2}$ peak gives two contributions with binding energy of 854.6 (low intensity) and $855.5 \mathrm{eV}$ (dominant). While the latter can be attributed to $\mathrm{Ni}-\mathrm{N}$ bonds in pseudooctahedral tetraazamacrocyclic cations with hexacoordinated $\mathrm{Ni}(\mathrm{II})$ [54], the lower-intensity component might be indicative of the presence of impurities of starting complexes $[\mathrm{Ni}(\text { cyclam })]^{2+}$ and $[\mathrm{Ni}(\text { tet } b)]^{2+}$ with square-planar coordination $[54,55]$.

A rough estimate of the content of tetraazamacrocyclic complexes in functionalized GO samples was obtained from TGA curves (Figure 4). The main qualitative difference between GO samples before and after functionalization is the disappearance of a well-manifested weight loss step approximately from 100 to $200{ }^{\circ} \mathrm{C}$; this change apparently results from the conversion of $\mathrm{COOH}$ groups into anionic carboxylate species. The further, most important temperature interval at about 
$200-500{ }^{\circ} \mathrm{C}$ corresponds to combustion of the major carbonaceous fraction, including macrocyclic moieties attached. The contribution of tetraazamacrocyclic ligand organics (without $\mathrm{Ni}$, which remains in the ash) estimated from the weight difference at $350{ }^{\circ} \mathrm{C}$ for $\mathrm{GO}$ before and after functionalization is about $4 \%$ for both nanohybrids. The molecular masses of cyclam and tet $b$ ligands are 200 and $284 \mathrm{Da}$, respectively, which suggests that the molar content of tetraazamacrocyclic complexes is notably higher in $\mathrm{GO}+[\mathrm{Ni}($ cyclam $)]$ than in $\mathrm{GO}+[\mathrm{Ni}($ tet $b)]$. The coresponding elemental Ni content was calculated to be about 1.2 and $0.8 \%$, respectively.

Even though the above TGA-derived values can be considered very rough, they do not differ significantly from the data obtained by conventional elemental analysis of nickel content (EA), which are $1.87 \%$ for $\mathrm{GO}+[\mathrm{Ni}($ cyclam $)]$ and $1.10 \%$ for $\mathrm{GO}+[\mathrm{Ni}($ tet $b)]$. Furthermore, they are remarkably close to the ones obtained by a third independent technique, namely, by SEM-coupled energy dispersive X-ray spectroscopy (Figure 5). The values presented in Figure 5 refer to both weight and atomic Ni content measured at three different sites of each functionalized sample; in particular, the weight content comprises the range of $1.18-1.98 \%$ for $\mathrm{GO}+[\mathrm{Ni}(\mathrm{cyclam})]$ and of $0.91-$ $2.30 \%$ for $\mathrm{GO}+[\mathrm{Ni}($ tet $b)]$, the lower limits practically coincide with the TGA-derived values. On the other hand, the upper limit value in the case of $\mathrm{GO}+[\mathrm{Ni}($ cyclam $)]$ is very close to the value determined by conventional EA (i.e., 1.98 vs. $1.87 \%$ ), being overestimated (2.30\%) for GO+[Ni(tet b)] by a factor of 2 to 3 as compared to the Ni content obtained from EA (1.10\%) and TGA (0.8\%), respectively.

By using the model structure shown in Scheme 2, one can get an approximate estimate of the distribution of tetraazamacrocyclic moieties throughout GO sheets. This model (brutto formula $\mathrm{C}_{148} \mathrm{H}_{46} \mathrm{O}_{23}$ ) has a molecular weight of $2192 \mathrm{Da}$ and is roughly $2 \mathrm{~nm}$ across. In the case of functionalization with [Ni(cyclam) $]\left(\mathrm{ClO}_{4}\right)_{2}$ according to Scheme 2, the substitution of all six carboxylic protons with $[\mathrm{Ni}($ cyclam $)] \mathrm{OH}$ monocations gives the product 
$\mathrm{C}_{148} \mathrm{H}_{40} \mathrm{O}_{23}+6\left(\mathrm{C}_{10} \mathrm{H}_{24} \mathrm{~N}_{4} \mathrm{Ni}(\mathrm{OH})\right)$, whose molecular weight is $3840 \mathrm{Da}$, and $\mathrm{Ni}$ content is about $9.2 \%$. The latter value is roughly one order of magnitude higher than the Ni content estimates from TGA, EA and EDS. A square model built of one hundred such GO fragments (10×10 arrangement) would have only 60 tetraazamacrocyclic complexes attached, that is, the Ni content would decrease by one order of magnitude approaching the experimentally derived values. The $10 \times 10$ model would be of ca. $20 \mathrm{~nm}$ across: such small sheets can be found in large numbers in most GO samples, along with more extended ones with a size of the order of $10^{2}-10^{3} \mathrm{~nm}$. One should also remember about the holes within $\mathrm{GO}$ sheets, where reactive $\mathrm{COOH}$ groups exist, as well as the fact that the chemical model in Scheme 2 is merely simplistic and accounts for carboxyls at two of four edges only, and on the contrary, does not account for a large fraction of carboxylates which remain uncoordinated.

Comparative morphological characterization of GO before and after functionalization with tetraazamacrocyclic complexes was performed by two electron (SEM and TEM) and two scanning probe (AFM and STM) microscopy techniques. As observed by conventional SEM (Figure 6), which provides the lowest resolution of all the microscopies employed, both GO and its macrocyclic nanohybrids exhibit numerous folds, crinkles, and rolled edges. Nevertheless, these features become more pronounced in the case of $\mathrm{GO}+[\mathrm{Ni}($ tet $b)]$ and especially $\mathrm{GO}+[\mathrm{Ni}($ cyclam)]; similar effects of functionalization with porphyrin tetraazamacrocyclic complexes on SEM morphology were reported elsewhere [16]. Finer morphological details were revealed by TEM. While single GO sheets before functionalization have relatively straight and smooth edges (Figure 7a,d), the latter become irregularly shaped and crinkled in the case of $\mathrm{GO}+[\mathrm{Ni}($ cyclam) $]$ (Figure 7b,e) and $\mathrm{GO}+[\mathrm{Ni}($ tet $b)]$ (Figure 7c,f). Similar morphological effects were observed in TEM images of GO sample edges amide-functionalized with porphyrins [10] and axial metal coordination-functionalized with porphyrins and phthalocyanines [17]. The likely reason for these changes is that bulky and rigid tetraazamacrocyclic molecules distort planar GO sheets at the points of functionalization attachment. 
The latter interpretation is supported by the analysis of AFM (Figure 8) and STM images (Figure 9), which reveal the appearance of grainy texture (and folding of some sheets) in functionalized GO samples, similarly to earlier AFM observations of GO functionalized with porphyrins [13].

The main goal of the present study was to verify whether coordination attachment of $[\mathrm{Ni}(\text { cyclam })]^{2+}$ and $[\mathrm{Ni}(\text { tet } b)]^{2+}$ tetraazamacrocyclic cations (whose perchlorates used as starting reagents are diamagnetic) to carboxylic groups of GO can result in the generation of paramagnetic properties in GO. The first attempt to detect paramagnetism was made by using EPR spectroscopy. All the spectra obtained (Figure S4 of Supporting Information) are dominated by the line with $g$ factor at about 2.003 typical for GO and commonly found for carbon-related dangling bonds [5659]. In addition, the spectrum of GO before functionalization exhibits characteristic $\mathrm{Mn}^{+2}(S=5 / 2, I$ $=5 / 2$ ) hyperfine sextet pattern centered close to $g=2.002$, which was attributed [59-61] to Mn impurities that originate from using $\mathrm{KMnO}_{4}$ as an oxidizing agent for $\mathrm{GO}$ preparation. Interestingly, the sextet disappeared after the treatment with tetraazamacrocyclic complexes: apparently, the basic reaction medium can efficiently wash out the manganese impurities. On the other hand, no new EPR signals were detected due to paramagnetic $\mathrm{Ni}^{2+}$ ions. This observation, however, does not disprove the coordination attachment of $[\mathrm{Ni}(\text { cyclam })]^{2+}$ and $[\mathrm{Ni}(\text { tet } b)]^{2+}$ cations to $\mathrm{COOH}$ groups of GO, as non-Kramers transition metal ions with integer-spin ground states $(S=1$ in our particular case of six-coordinated $\mathrm{Ni}(\mathrm{II})$ ) remain EPR-silent at conventional frequencies and mode of data collection [62] (perpendicular mode X-band EPR was used in the present work).

In order to confirm whether spin state of the Ni(II) centers changed in the course of depositing the macrocycles onto GO, room temperature magnetic susceptibility measurements were performed using portable Evans magnetic balance (Table S1 of Supporting Information). Although the accuracy and precision of these measurements are not high, the results still clearly distinguish diamagnetic samples from the paramagnetic ones, and provide a useful estimate of the average 
number of unpaired electrons per metal ions. As expected, starting samples of GO were diamagnetic. Furthermore, both tetraazamacrocyclic complexes used in this work, $[\mathrm{Ni}($ cyclam $)]\left(\mathrm{ClO}_{4}\right)_{2}$ and $[\mathrm{Ni}($ tet $b)]\left(\mathrm{ClO}_{4}\right)_{2}$, are also diamagnetic: strong equatorial ligand field of tetraazamacrocycles is known to support low spin state of the square planar Ni(II) complexes in the presence of poorly coordinating anions (perchlorate, in the present case) [63]. However, their deposition onto the surface of GO afforded notably paramagnetic samples. Although the values of gram susceptibility for the functionalized samples appeared to be quite low, relatively low nickel content in them (ca. $1 \%$ by mass) should be taken into account. Analytical data for both samples allowed us to estimate molar susceptibility per nickel and evaluate the effective magnetic moments of the tetraazamacrocycles deposited: $1.95 \mathrm{BM}$ and $2.2 \mathrm{BM}$ for $\mathrm{GO}+[\mathrm{Ni}($ cyclam $)]$ and $\mathrm{GO}+[\mathrm{Ni}($ tet $b)$, respectively. These values are somewhat lower than, but generally close to, the spin-only values for high spin $\mathrm{Ni}$ (II) (2.83 BM for two unpaired electrons). We did not take into account significant diamagnetic corrections for these composite samples, which are partly responsible for an apparent decrease in effective magnetic moments of nickel. While more accurate measurements would be needed to fully analyze magnetic behavior of nickel-modified carbon nanomaterials, the results obtained indicate changes fully consistent with the targeted low-spin to high-spin transition of the $\mathrm{Ni}(\mathrm{II})$ upon coordination functionalization of GO carboxyls with $\left[\mathrm{Ni}(\text { cyclam) }]^{2+}\right.$ and $[\mathrm{Ni}(\text { tet } b)]^{2+}$ cations.

The above magnetic susceptibility measurements detected no evident ferromagnetic coupling with/through GO, that is, no drastic increase in magnetic moment beyond that expected for axial coordination was observed. For a deeper insight into magnetic properties of graphene-based nanohybrids with tetraazamacrocyclic complexes, the use of theoretical tools such as DFT calculations can be useful, as was previously demonstrated for porphyrins [12]. In an attempt to provide details of electronic structure and interactions in $\mathrm{GO}+[\mathrm{Ni}($ cyclam $)]$ and $\mathrm{GO}+[\mathrm{Ni}($ tet $b)]$ 
systems, we employed DFT calculations by using PBE GGA functional with Grimme dispersion correction in conjunction with DNP basis set; this combination was found to be useful and efficient to study the interaction of $\mathrm{Ni}(\mathrm{II})$ macrocyclic complexes with $\pi$-conjugated nanocarbons [42-44]. The studies on such extended systems, especially when they include transition metal atoms, are known to be highly computationally demanding, being prohibitive for GO models of the size comparable with that of GO sheet shown in Scheme 2. Any additional oxidized functionality increases considerably the conformational freedom, and thus creates additional convergence problems. Therefore, we limited the GO model of choice to a relatively rigid ten aromatic-ring system, in which dangling bonds at the edge were terminated with eight carbonyl $\mathrm{O}$ atoms and six carboxylic functionalities, as shown in Figure 10. In order to simulate the coordination attachment of tetraazamacrocyclic cations of $\mathrm{Ni}(\mathrm{II})$ in a basic medium, all the carboxylic groups were deprotonated, resulting in the total charge of 6- for this GO model. The presence of six carboxylates creates notable distortion (edge view of the optimized GO geometry in Figure 10) of otherwise planar graphene sheet. This distortion remains the same after coordination of $[\mathrm{Ni}(\mathrm{cyclam})]^{2+}$ cation, but further increases when $[\mathrm{Ni}(\text { tet } b)]^{2+}$ cation is attached to the GO model (edge views of the optimized geometries in Figure 10). The likely reason is that tet $b$ ligand having six methyl substituents is more bulky than cyclam, and therefore causes stronger interference with GO geometry. The calculated formation energies for tetraanionic $\mathrm{GO}+[\mathrm{Ni}($ cyclam $)]$ and $\mathrm{GO}+[\mathrm{Ni}($ tet $b)]$ complexes are very high, with absolute values of 619.78 and $613.22 \mathrm{kcal} / \mathrm{mol}$, respectively (the negative values in Table 1 imply highly exothermic process), resulting from strong attraction of the macrocyclic cations to the negatively charged GO model. The newly formed $\mathrm{Ni}-\mathrm{O}$ coordination bonds are 2.038 and $2.129 \AA$ for $\mathrm{GO}+[\mathrm{Ni}($ cyclam)], 2.010 and $2.227 \AA$ for $\mathrm{GO}+[\mathrm{Ni}($ tet $b)]$; for comparison, the corresponding distances in the crystalline $[\mathrm{Ni}(\text { tet } b)]^{2+}$ nicotinate complex were found to be almost equal, 2.153 and $2.156 \AA$ [32], and slightly differing in its bridged 
pyridinedicarboxylate analogue, 2.123 and $2.255 \AA$ [31]. The non-equivalence of $\mathrm{Ni}-\mathrm{O}$ distances was attributed to steric hindrance between one $[\mathrm{Ni}(\operatorname{tet} b)]^{2+}$ cation and the remaining part of the bridged complex [31]; apparently, similar factors act in the present case of GO tetraazamacrocyclic nanohybrids.

The structure of frontier orbitals HOMO and LUMO (Figure 10) changes to a different degree for different coordinated tetraazamacrocyclic complexes. The shape of HOMO undergoes similar changes in both cases, where part of it slightly extends to the Ni atom and considerably reduces in size on the coordinated $\mathrm{COO}^{-}$group and its neighboring atoms. The shape of LUMO in the case of $\mathrm{GO}+[\mathrm{Ni}($ tet $b)]$ remains essentially unchanged as compared to $\mathrm{GO}$, whereas for $\mathrm{GO}+\left[\mathrm{Ni}(\right.$ cyclam) $]$, the lobes extend significantly to the $\mathrm{NiN}_{4} \mathrm{O}_{2}$ coordination sphere and reduce in size at one side of GO sheet. This observation correlates with a stronger interaction (by about 6.5 $\mathrm{kcal} / \mathrm{mol})$ in the case of $\mathrm{GO}+[\mathrm{Ni}($ cyclam $)]$. In terms of HOMO-LUMO gap, the values calculated for GO tetraazamacrocyclic nanohybrids, which are 0.199 and $0.203 \mathrm{eV}$ for $\mathrm{GO}+[\mathrm{Ni}($ cyclam)] and $\mathrm{GO}+[\mathrm{Ni}($ tet $b)]$, respectively, are even lower than the one calculated for $\mathrm{GO}(0.429 \mathrm{eV})$. This can be interpreted as the enhancement of conductivity properties of GO resulting from the coordination functionalization with $[\mathrm{Ni}(\text { cyclam })]^{2+}$ and $[\mathrm{Ni}(\text { tet } b)]^{2+}$ cations.

Finally, we analyzed spin density distribution in $\mathrm{GO}+[\mathrm{Ni}($ cyclam $)]$ and $\mathrm{GO}+[\mathrm{Ni}($ tet $b)]$; the corresponding plots are presented in Figure 10 (right column). As was expected, the main spin density is concentrated at the paramagnetic $\mathrm{Ni}(\mathrm{II})$ centers along with all $\mathrm{N}$ and $\mathrm{O}$ donor atoms. It can also be found on other atoms of the macrocyclic ligands, especially on those of cyclam. In addition, a number of smaller lobes appears on some $\mathrm{C}$ atoms of graphene sheet, as well as on $\mathrm{O}$ atoms of carbonyl and $\mathrm{COO}^{-}$groups. This favors the presence of some ferromagnetic coupling in $\mathrm{GO}+[\mathrm{Ni}($ cyclam $)]$ and $\mathrm{GO}+[\mathrm{Ni}($ tet $b)]$ nanohybrids, though its degree is not significant, as the results of magnetic susceptibility measurements suggest. 


\section{Conclusions}

Coordination attachment of $[\mathrm{Ni}(\mathrm{cyclam})]^{2+}$ and $[\mathrm{Ni}(\text { tet } b)]^{2+}$ tetraazamacrocyclic cations to carboxylic groups of GO takes place under basic conditions in aqueous-based reaction medium. The procedure is very facile and does not require high temperatures or other harsh conditions. Changing the coordination geometry of $\mathrm{Ni}(\mathrm{II})$ from square-planar tetracoordinated to pseudooctahedral hexacoordinated is accompanied by the conversion from low-spin to high-spin metal centers. Even though the content of tetraazamacrocyclic complexes in functionalized GO samples is relatively low (nickel content of ca. $1 \mathrm{wt} \%$ as determined by TGA, EA and EDS), room temperature magnetic susceptibility measurements easily detected the appearance of paramagnetic properties in $\mathrm{GO}+[\mathrm{Ni}($ cyclam $)]$ and $\mathrm{GO}+[\mathrm{Ni}($ tet $b)]$ nanohybrids, with effective magnetic moments of $1.95 \mathrm{BM}$ and 2.2 BM, respectively. The coordination attachment of $\mathrm{Ni}(\mathrm{II})$ tetraazamacrocycles results in considerable changes in FTIR and XPS spectra, as well as in GO morphology, as observed by SEM, TEM, AFM and STM. The proposed functionalization approach allows for a facile generation of paramagnetic centers in GO starting from two diamagnetic components.

\section{Acknowledgements}

Financial support from the Universidad Nacional Autónoma de México (UNAM; grants DGAPA-IN100815 and IN101313) and the National Council of Science and Technology of Mexico (CONACyT; grant 251164) is greatly appreciated. N. A.-C. and L. V. H.-H. are indebted to the Doctorate Program in Chemical Sciences of UNAM and CONACyT for a PhD fellowship. E. V. R.A. is grateful to the NSF for partial financial support (grants CHE1412909 and CHE1229426). The authors are grateful to Iván Puente-Lee (Facultad de Química, UNAM) for TEM imaging; to Dr. Victor Meza-Laguna (Instituto de Ciencias Nucleares, UNAM), for SEM imaging and EDS 
measurements; to Alejandra Moreno-Bárcenas and Dr. J. Francisco Pérez-Robles (Centro de Investigación y de Estudios Avanzados, Instituto Politécnico Nacional), for XPS spectra acquisition. We also thank Dr. Edgar Alvarez Zauco (Facultad de Ciencias, UNAM) for the use of TGA equipment. E. V. B. and V. A. B. are on sabbatical leave from UNAM; E. V. B. appreciates the support from CONACyT (grant 250604). 


\section{References}

1. V. Georgakilas, M. Otyepka, A. B. Bourlinos, V. Chandra, N. Kim, K. C. Kemp, P. Hobza, R. Zboril, K. S. Kim, Functionalization of graphene: Covalent and non-covalent approaches, derivatives and applications, Chem. Rev. 112 (2012) 6156-6214.

2. D. Chen, H. Feng, J. Li, Graphene oxide: Preparation, functionalization, and electrochemical applications, Chem. Rev. 112 (2012) 6027-6053.

3. S. Niyogi, E. Bekyarova, J. Hong, S. Khizroev, C. Berger, W. de Heer, R. C. Haddon, Covalent chemistry for graphene electronics, J. Phys. Chem. Lett. 2 (2011) 2487-2498.

4. M. R. Axet, O. Dechy-Cabaret, J. Durand, M. Gouygou, P. Serp, Coordination chemistry on carbon surfaces, Coord. Chem. Rev. 308, 236-345 (2016).

5. J. Malig, N. Jux, D. M. Guldi, Toward multifunctional wet chemically functionalized grapheneIntegration of oligomeric, molecular, and particulate building blocks that reveal photoactivity and redox activity, Acc. Chem. Res. 46 (2013) 53-64.

6. I. V. Lightcap, P. V. Kamat, Graphitic design: Prospects of graphene-based nanocomposites for solar energy conversion, storage, and sensing, Acc. Chem. Res. 46 (2013) 2235-2243.

7. A. N. Khlobystov, A. Hirsch, Organometallic and coordination chemistry of carbon nanomaterials, Dalton Trans. 43 (2014) 7345-7345.

8. A. Wojcik, P. V. Kamat, Reduced graphene oxide and porphyrin. An interactive affair in 2-D, ACS Nano 4 (2010) 6697-6706.

9. V. D. Pham, J. Lagoute, O. Mouhoub, F. Joucken, V. Repain, C. Chacon, A. Bellec, Y. Girard, S. Rousset, Electronic interaction between nitrogen-doped graphene and porphyrin molecules, ACS Nano 8 (2014) 9403-9409. 
10. J. Tang, L. Niu, J. Liu, Y. Wang, Z. Huang, S. Xie, L. Huang, Q. Xu, Y. Wang, L. A. Belfiore, Effect of photocurrent enhancement in porphyrin-graphene covalent hybrids, Mater. Sci. Eng. C 34 (2014) 186-192.

11. I. Kruusenberg, J. Mondal, L. Matisen, V. Sammelselg, K. Tammeveski, Oxygen reduction on graphene-supported $\mathrm{MN}_{4}$ macrocycles in alkaline media, Electrochem. Commun. 33 (2013) 18-22. 12. C. F. Hermanns, K. Tarafder, M. Bernien, A. Krüger, Y. M. Chang, P. M. Oppeneer, W. Kuch, Magnetic coupling of porphyrin molecules through graphene, Adv. Mater. 25 (2013) 3473-3477.

13. M. Zhu, Z. Li, B. Xiao, Y. Lu, Y. Du, P. Yang, X. Wang, Surfactant assistance in improvement of photocatalytic hydrogen production with the porphyrin noncovalently functionalized graphene nanocomposite, ACS Appl. Mater. Interfaces 5 (2013) 1732-1740.

14. P. Guo, P. Chen, M. Liu, One-dimensional porphyrin nanoassemblies assisted via graphene oxide: Sheetlike functional surfactant and enhanced photocatalytic behaviors, ACS Appl. Mater. Interfaces 5 (2013) 5336-5345.

15. J. Geng, H.-T. Jung, Porphyrin functionalized graphene sheets in aqueous suspensions: From the preparation of graphene sheets to highly conductive graphene films, J. Phys. Chem. C 114 (2010) 8227-8234.

16. D. Huang, J. Lu, S. Li, Y. Luo, C. Zhao, B. Hu, M. Wang, Y. Shen, Fabrication of cobalt porphyrin - electrochemically reduced graphene oxide hybrid films for electrocatalytic hydrogen evolution in aqueous solution, Langmuir 30 (2014) 6990-6998.

17. M. Jurow, V. Manichev, C. Pabon, B. Hageman, Y. Matolina, C. M. Drain, Self-organization of $\mathrm{Zr}(\mathrm{IV})$ porphyrinoids on graphene oxide surfaces by axial metal coordination, Inorg. Chem. 52 (2013) 10576-10582.

18. A. Wang, L. Long, W. Zhao, Y. Song, M. G. Humphrey, M. P. Cifuentes, X. Wu, Y. Fu, 
D. Zhang, X. Li, C. Zhang, Increased optical nonlinearities of graphene nanohybrids covalently functionalized by axially-coordinated porphyrins, Carbon 533 (2013) 327-338 .

19. S. Lisi, P. Gargiani, M. Scardamaglia, N. B. Brookes, V. Sessi, C. Mariani, M. G. Betti, Graphene-induced magnetic anisotropy of a two-dimensional iron phthalocyanine network, J. Phys. Chem. Lett. 6 (2015) 1690-1695.

20. M. Bernien, H. Naggert, L. M. Arruda, L. Kipgen, F. Nickel, J. Miguel, C. F. Hermanns, A. Krüger, D. Krüger, E. Schierle, E. Weschke, F. Tuczek, W. Kuch, Highly efficient thermal and light-induced spin-state switching of an Fe(II) complex in direct contact with a solid surface, ACS Nano 9 (2015) 8960-8966.

21. Y. Murashima, M. R. Karim, N. Saigo, H. Takehira, R. Ohtani, M. Nakamura, M. Koinuma, L. F. Lindoy, K. Kuroiwad, S. Hayami, Graphene oxide and reduced graphene oxide hybrids with spin crossover iron(III) complexes, Inorg. Chem. Front. 2 (2015) 886-892

22. C. F. Hermanns, K. Tarafder, M. Bernien, A. Krüger, Y.-M. Chang, P. M. Oppeneer, W. Kuch, Magnetic coupling of porphyrin molecules through graphene, Adv. Mater. 25 (2013) 3473-3477.

23. S. Bhandary, O. Eriksson, B. Sanyal, Defect controlled magnetism in FeP/graphene/Ni(111), Sci. Rep. 3 (2013) 3405(1-6).

24. D. Klar, S. Bhandary, A. Candini, L. Joly, P. Ohresser, S. Klyatskaya, M. Schleberger, M. Ruben, M. Affronte, O. Eriksson, B. Sanyal, H. Wende, Field-regulated switching of the magnetization of Co-porphyrin on graphene, Phys. Rev. B 89 (2014) 144411(1-5).

25. J. Mao, H. Zhang, Y. Jiang, Y. Pan, M. Gao, W. Xiao, H.-J. Gao, Tunability of supramolecular kagome lattices of magnetic phthalocyanines using graphene-based moiré patterns as templates, $\mathrm{J}$. Am. Chem. Soc. 131 (2009) 14136-14137.

26. A. Candini, V. Bellini, D. Klar, V. Corradini, R. Biagi, V. De Renzi, K. Kummer, N. B. Brookes, U. del Pennino, H. Wende, M. Affronte, Ferromagnetic exchange coupling between Fe 
phthalocyanine and $\mathrm{Ni}(111)$ surface mediated by the extended states of graphene, J. Phys. Chem. C 118 (2014) 17670-17676.

27. A. Candini, S. Klyatskaya, M. Ruben, W. Wernsdorfer, M. Affronte, Graphene spintronic devices with molecular nanomagnets, Nano Lett. 11 (2011) 2634-2639.

28. A. H. Hung, R. J. Holbrook, M. W. Rotz, C. J. Glasscock, N. D. Mansukhani, K. W. MacRenaris, L. M. Manus, M. C. Duch, K. T. Dam, M. C. Hersam, T. J. Meade, Graphene oxide enhances cellular delivery of hydrophilic small molecules by co-incubation, ACS Nano 8 (2014) 10168-10177.

29. X. Liang, P. J. Sadler, Cyclam complexes and their applications in medicine, Chem. Soc. Rev. 33 (2004) 246-266.

30. P. O. Whimp, M. F. Bailey, N. F. Curtis, Some cyclic tetra-amines and their metal-ion complexes. Part VI. The crystal structure of acetato-C-rac-(5,7,7,12,14,14-hexamethyl-1,4,8,11tetra-azacyclotetradecane)nickel(II) perchlorate, J. Chem. Soc. A (1970) 1956-1963.

31. E. V. Basiuk, V. A. Basiuk, J. Gómez-Lara, R. A. Toscano, A bridged high-spin complex bis[Ni(II)(rac-5,5,7,12,12,14-hexamethyl-1,4,8,11-tetraazacyclotetradecane)]-2,5pyridinedicarboxylate diperchlorate monohydrate, J. Inclusion Phenom. Macrocyclic Chem. 38 (2000) 45-56.

32. E. V. Basiuk, V. A. Basiuk, S. Hernández-Ortega, M. Martínez-García, J.-M. Saniger-Blesa, (Crac-5,5,7,12,12,14-Hexamethyl-1,4,8,11-tetraazacyclotetradecane- $\left.\kappa^{4} N\right)\left(\right.$ nicotinato- $\left.O, O^{\prime}\right)$ nickel(II) perchlorate, Acta Crystallogr. C C57 (2001) 553-555.

33. R. W. Hay, G. A. Lawrance, N. F. Curtis, A convenient synthesis of the tetra-aza-macrocyclic ligands trans-[14]-diene, tet $a$, and tet $b$, J. Chem. Soc. Perkin Trans. 1 (6) (1975) 591-593. 
34. A. M. Tait, D. H. Busch, 5,5,7,12,12,14-Hexamethyl-1,4,8,11-tetraazacyclotetradecane $\left(5,5,7,12,12,14-\mathrm{Me}_{6}[14]\right.$ ane-1,4,8,11-N 4$)$ complexes, in Inorganic Syntheses, Vol. 18, ed. B. E. Douglas, Wiley, New York, 1978, pp. 10-15.

35. E. K. Barefield, F. Wagner, A. W. Herlinger, A. R. Dahl, S. Holt, (1,4,8,11Tetraazacyclotetradecane)Nickel(II) perchlorate and 1,4,8,11-tetraazacyclotetradecane, in Inorganic Syntheses, Vol. 16, ed. F. Basolo, Wiley, New York, 1976, pp. 220-225.

36. B. Delley, An all-electron numerical method for solving the local density functional for polyatomic molecules, J. Chem. Phys. 92 (1990) 508-517.

37. B. Delley, Fast calculations of electrostatics in crystals and large molecules, J. Phys. Chem. 100 (1996) 6107-6110.

38. B. Delley, From molecules to solids with the DMol3 approach, J. Chem. Phys. 113 (2000) 77567764.

39. B. Delley, D. E. Ellis, A. J. Freeman, E. J. Baerends, D. Post, Binding energy and electronic structure of small copper particles, Phys. Rev. B 27 (1983) 2132-2144.

40. J. P. Perdew, K. Burke, M. Ernzerhof, Generalized gradient approximation made simple, Phys. Rev. Lett. 77 (1996) 3865-3568.

41. S. Grimme, Semiempirical GGA-type density functional constructed with a long-range dispersion correction, J. Comput. Chem. 27 (2006) 1787-1799.

42. L. V. Henao-Holguín, V. A. Basiuk, Interaction of a Ni(II) tetraazaannulene complex with elongated fullerenes as simple models for carbon nanotubes, J. Mol. Model. 21 (2015) 146(1-11). 43. V. A. Basiuk, L. V. Henao-Holguín, Dispersion-corrected density functional theory calculations of meso-tetraphenylporphine- $\mathrm{C}_{60}$ complex by using DMol3 module, J. Comput. Theor. Nanosci. 11 (2014) 1609-1615. 
44. E. V. Basiuk, M. Martínez-Herrera, E. Álvarez-Zauco, L. V. Henao-Holguín, I. Puente-Lee, V. A. Basiuk, Noncovalent functionalization of graphene with a Ni(II) tetraaza[14]annulene complex, Dalton Trans. 43 (2014) 7413-7428.

45. S. Yang, B. Deng, R. Ge, L. Zhang, H. Wang, Z. Zhang, W. Zhu, G. Wang, Electrodeposition of porous graphene networks on nickel foams as supercapacitor electrodes with high capacitance and remarkable cyclic stability, Nanoscale Res. Lett. 9 (2014) 672(1-11).

46. V. N. Nemykin, P. Galloni, B. Floris, C. D. Barrett, R. G. Hadt, R. I. Subbotin, A. G. Marrani, R. Zanoni, N. M. Loim, Metal-free and transition-metal tetraferrocenylporphyrins. Part 1: Synthesis, characterization, electronic structure, and conformational flexibility of neutral compounds, Dalton Trans. (32) (2008) 4233-4246.

47. Y. Niwa, H. Kobayashi, T. Tsuchiya, X-Ray photoelectron spectroscopy of azaporphyrins, Inorg. Chem. 13 (1974) 2891-2896.

48. P. G. Gassman, A. Ghosh, J. Almlöf, Electronic effects of peripheral substituents in porphyrins: X-ray photoelectron spectroscopy and ab initio self-consistent field calculations, J. Am. Chem. Soc. 114 (1992) 9990-10000.

49. M. Seno, S. Tsuchiya, S. Ogawa, Electronic structure of macrocyclic compounds revealed by Xray photoelectron spectroscopy, J. Am. Chem. Soc. 99 (1977) 3014-3018.

50. J. P. Macquet, M. M. Millard, T. Theophanides, X-Ray photoelectron spectroscopy of porphyrins, J. Am. Chem. Soc. 100 (1978) 4741-4746.

51. G. Zhou, D.-W. Wang, L.-C. Yin, N. Li, F. Li, H.-M. Cheng, Oxygen bridges between NiO nanosheets and graphene for improvement of lithium storage, ACS Nano 6 (2012) 3214-3223. 52. Z. Tan, W. Zhang, D. Qian, C. Cui, Q. Xu, L. Li, S. Li, Y. Li, Solution-processed nickel acetate as hole collection layer for polymer solar cells, Phys. Chem. Chem. Phys. 14 (2012) 14217-14223. 
53. K. Kishi, S. Ikeda, X-Ray photoelectron spectroscopic study for the adsorption of acetic acid and ethylenediamine on iron and nickel, Applications Surf. Sci. 5 (1980) 7-20.

54. G. J. Colpas, M. J. Maroney, C. Bagyinka, M. Kumar, W. S. Willis, S. L. Suib, P. K. Mascharak, N. Baidya, X-Ray spectroscopic studies of nickel complexes, with application to the structure of nickel sites in hydrogenases, Inorg. Chem. 30 (1991) 920-928.

55. L. J. Matienzo, L. I. Yin, S. O. Grim, W. E. Swartz, X-Ray photoelectron spectroscopy of nickel compounds, Inorg. Chem. 12 (1973) 2762-2769.

56. R. C. Barklie, Characterisation of defects in amorphous carbon by electron paramagnetic resonance, Diam. Relat. Mater. 10 (2001) 174-181.

57. J. Kausteklis, P. Cevc, D. Arčon, L. Nasi, D. Pontiroli, M. Mazzani, M. Riccò, Electron paramagnetic resonance study of nanostructured graphite, Phys. Rev. B 84 (2011) 125406(1-5). 58. S. S. Rao, A. Stesmans, D. V. Kosynkin, A. Higginbotham, J. M. Tour, Paramagnetic centers in graphene nanoribbons prepared from longitudinal unzipping of carbon nanotubes, New J. Phys. 13 (2011) 113004 (1-9).

59. C. V. Pham, M. Krueger, M. Eck, S. Weber, E. Erdem, Comparative electron paramagnetic resonance investigation of reduced graphene oxide and carbon nanotubes with different chemical functionalities for quantum dot attachment, Appl. Phys. Lett. 104 (2014) 132102(1-5).

60. A. M. Panich, A. I. Shamesa, A. E. Aleksenskii, A. Dideikin, Magnetic resonance evidence of manganese-graphene complexes in reduced graphene oxide, Solid State Comm. 152 (2012) 466468.

61. A. M. Panich, A. I. Shames, N. A. Sergeev, Paramagnetic impurities in graphene oxide, Appl. Magn. Reson. 44 (2013) 107-116. 
62. J. Krzystek, J.-H. Park, M. W. Meisel, M. A. Hitchman, H. Stratemeier, L.-C. Brunel, J. Telser, EPR Spectra from "EPR-silent” species: High-frequency and high-field EPR spectroscopy of pseudotetrahedral complexes of nickel(II), Inorg. Chem. 41 (2002) 4478-4487.

63. V. Amendola, L. Fabbrizzi, F. Foti, M. Licchelli, C. Mangano, P. Pallavicini, A. Poggi, D.

Sacchi, A. Taglietti, Light-emitting molecular devices based on transition metals, Coord. Chem. Rev. 250 (2006) 273-299. 
Table 1. Formation energies $\Delta E_{\mathrm{GO}+\mathrm{Ni}}($ in $\mathrm{kcal} / \mathrm{mol}$ ) for the high-spin complexes $\mathrm{GO}+[\mathrm{Ni}($ cyclam$)]$ and $\mathrm{GO}+[\mathrm{Ni}($ tet $b)]$ along with the respective HOMO, LUMO and HOMO-LUMO gap energies (in eV) and the Ni-O distances (in $\AA$ ) between carboxylic O and central Ni atoms, as well as HOMO, LUMO and HOMO-LUMO gap energies for GO and square-planar macrocyclic cations $[\mathrm{Ni}(\mathrm{cyclam})]^{2+}$ and $[\mathrm{Ni}(\text { tet } b)]^{2+}$. The DFT calculations were performed by using PBE GGA functional with Grimme dispersion correction in conjunction with DNP basis set. Optimized geometries for $\mathrm{GO}, \mathrm{GO}+[\mathrm{Ni}($ cyclam) $]$ and $\mathrm{GO}+[\mathrm{Ni}($ tet $b)]$ models along with $\mathrm{HOMO}$, LUMO, and spin density plots are shown in Figure 10.

\begin{tabular}{llllll}
\hline Compound & $\Delta E_{\mathrm{GO}+\mathrm{Ni}}, \mathrm{kcal} / \mathrm{mol}$ & $E_{\mathrm{HOMO}}, \mathrm{eV}$ & $E_{\mathrm{LUMO}}, \mathrm{eV}$ & $E_{\mathrm{gap}}, \mathrm{eV}$ & $\mathrm{Ni}-\mathrm{O}$ distance, $\AA$ \\
\hline $\mathrm{GO}^{a}$ & & & & \\
{$[\mathrm{Ni}(\mathrm{cyclam})]^{2+}$} & & -12.506 & -10.320 & 2.186 & \\
$\mathrm{GO}+[\mathrm{Ni}(\mathrm{cyclam})]^{a}$ & -619.78 & 3.901 & 4.100 & 0.199 & $2.038,2.129$ \\
{$[\mathrm{Ni}(\mathrm{tet} b)]^{2+}$} & -11.833 & -9.752 & 2.081 & \\
$\mathrm{GO}+[\mathrm{Ni}(\mathrm{tet} b)]^{a}$ & -613.22 & 3.787 & 3.990 & 0.203 & $2.010,2.227$ \\
\hline
\end{tabular}

${ }^{a}$ All the carboxylic groups are deprotonated, as in a basic medium, resulting in the total charge of 6- for GO model, and 4- for the complexes $\mathrm{GO}+[\mathrm{Ni}($ cyclam $)]$ and $\mathrm{GO}+[\mathrm{Ni}($ tet $b)]$. 

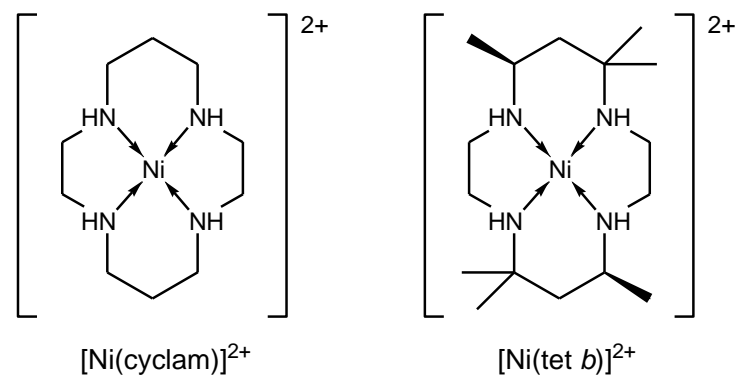

$[\mathrm{Ni}(\text { tet } b)]^{2+}$

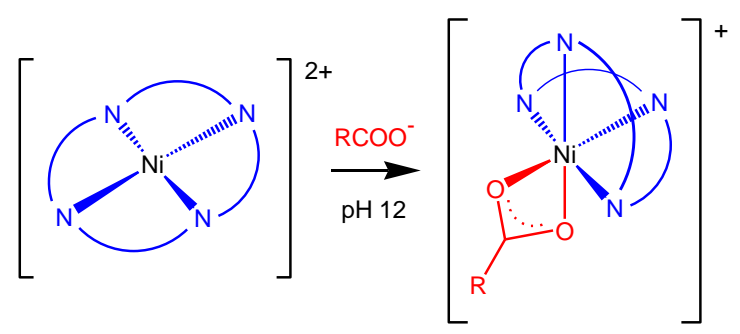

Scheme 1. Low-spin square planar tetraazamacrocyclic complexes of Ni(II) (top) and their conversion into respective high-spin octahedral carboxylates (bottom). 

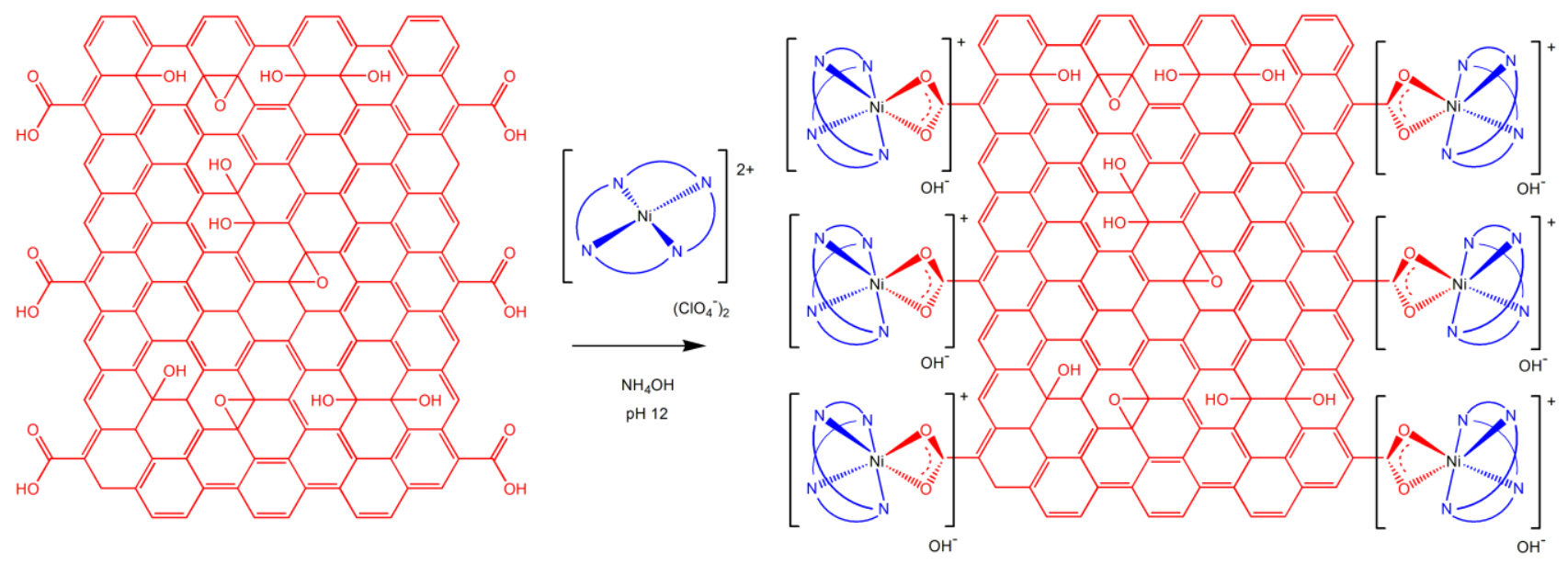

Scheme 2. Coordination functionalization of graphene oxide with Ni(II) tetraazamacrocyclic complexes via the formation of high-spin octahedral carboxylates with $\mathrm{COOH}$ groups at $\mathrm{GO}$ edges. GO model was adapted with some valency corrections from Tang et al. [10]. 


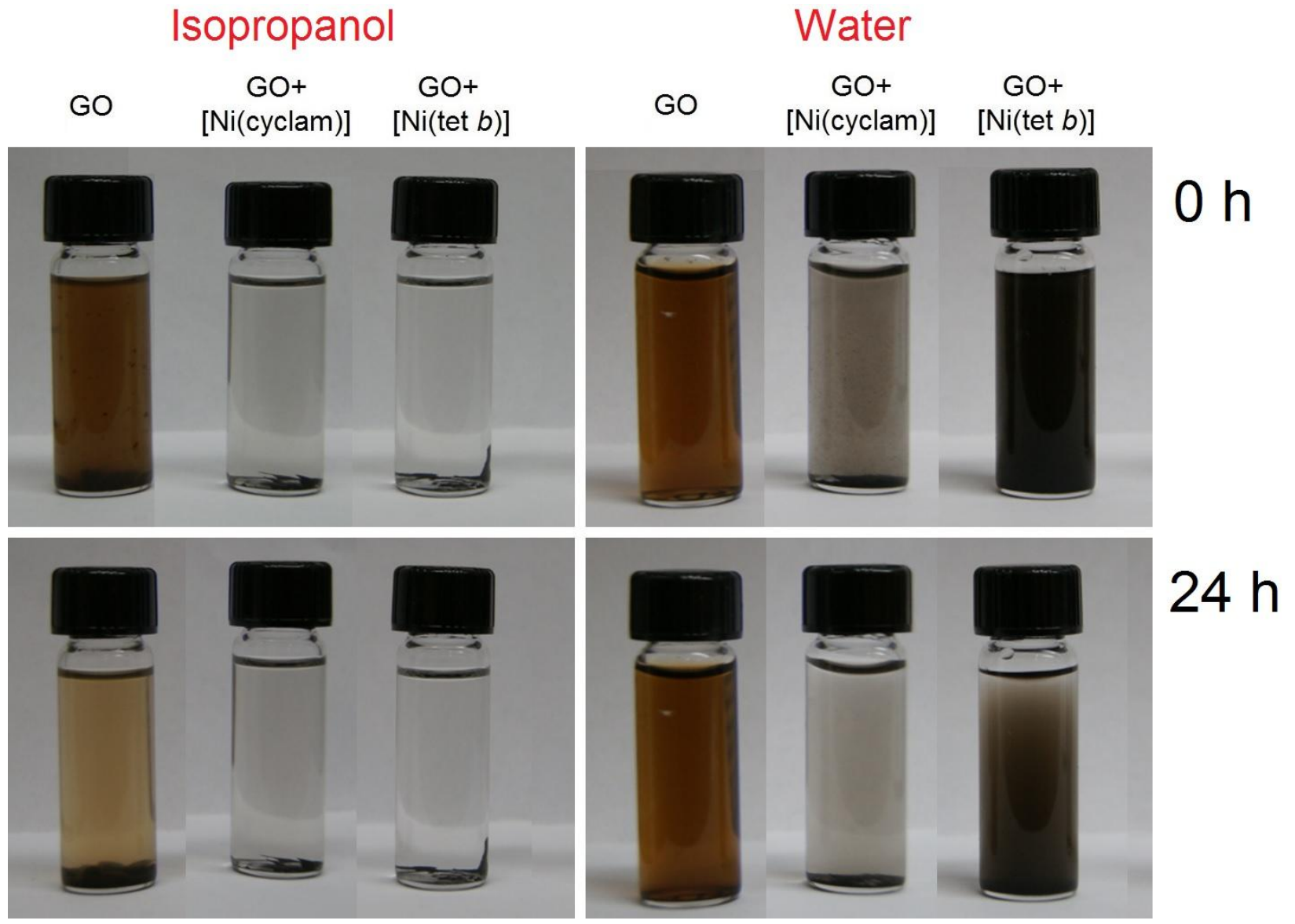

Figure 1. Solubility test ( $1 \mathrm{mg}$ of each sample per $2 \mathrm{ml}$ of solvent) in isopropanol and water for GO before and after coordination functionalization with $\mathrm{Ni}(\mathrm{II})$ tetraazamacrocyclic complexes. Images were taken at 0 and $24 \mathrm{~h}$ after ultrasound bath treatment for $5 \mathrm{~min}$. 


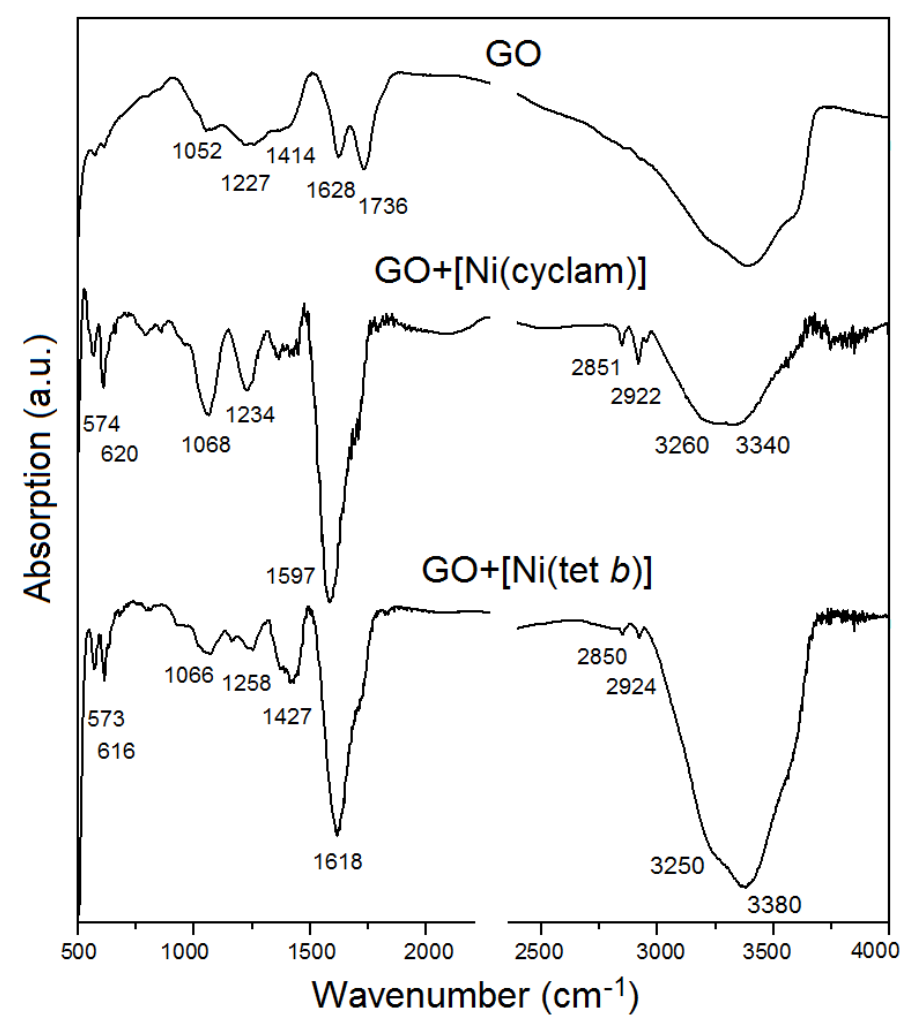

Figure 2. FTIR spectra of GO before and after coordination functionalization with $\mathrm{Ni}(\mathrm{II})$ tetraazamacrocyclic complexes. 

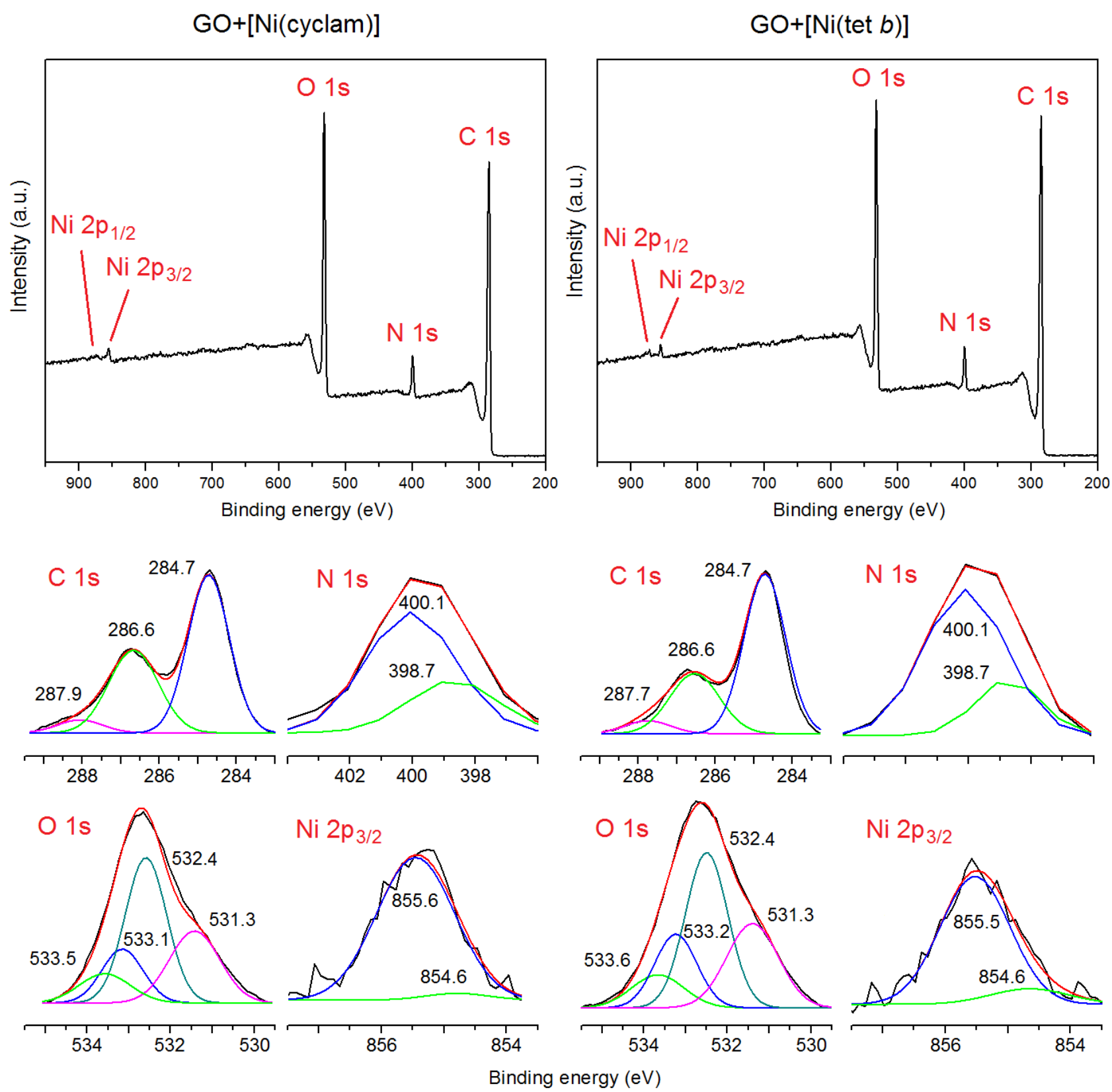

Binding energy (eV)

Figure 3. XPS spectra of GO samples functionalized with Ni(II) tetraazamacrocyclic complexes. Top: survey spectra of $\mathrm{GO}+[\mathrm{Ni}($ cyclam $)]$ and $\mathrm{GO}+[\mathrm{Ni}($ tet $b)]$; bottom: deconvolution of the corresponding C 1s, N 1s, O 1s and Ni 2p p/2 $_{2}$ lines. Raw spectra are shown in black, and sum, in red. 


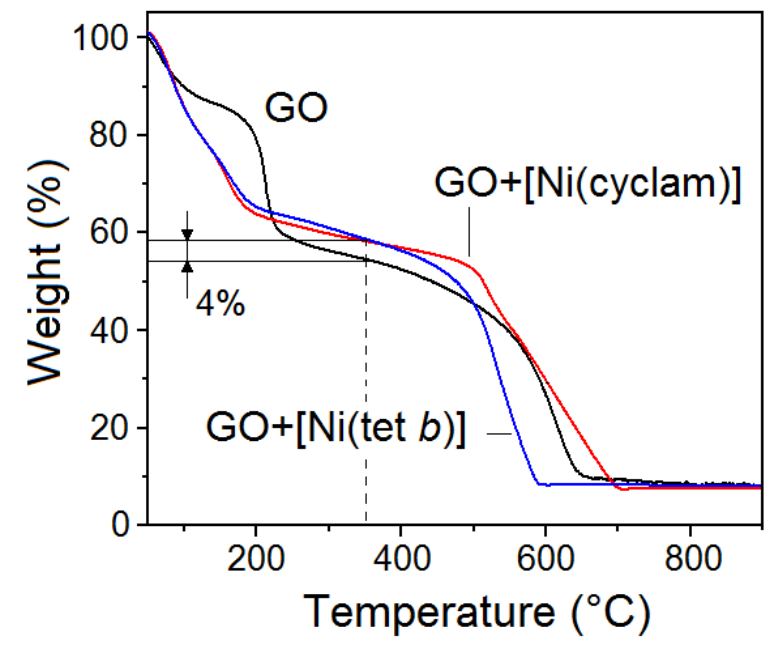

Figure 4. TGA curves for GO before and after coordination functionalization with $\mathrm{Ni}(\mathrm{II})$ tetraazamacrocyclic complexes. 

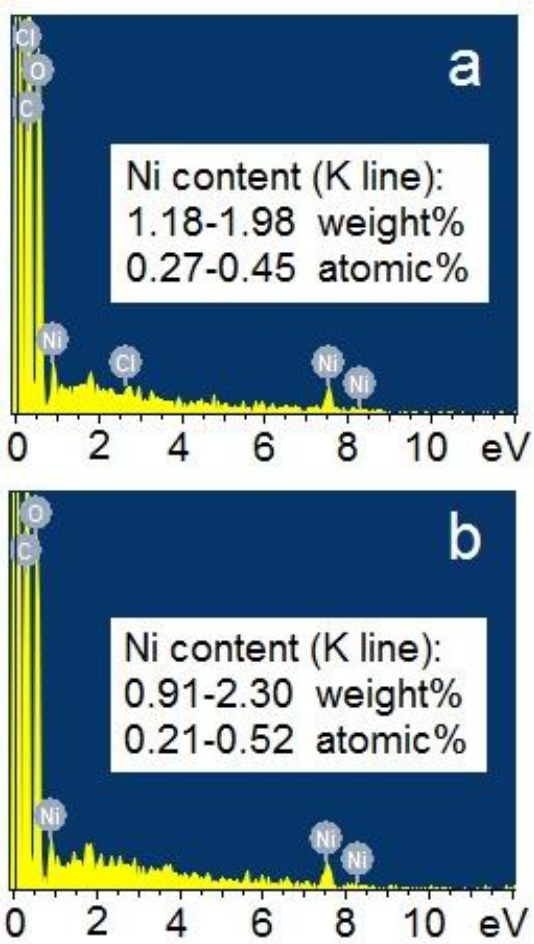

Figure 5. Representative EDS spectra of (a) $\mathrm{GO}+[\mathrm{Ni}($ cyclam) $]$ and (b) $\mathrm{GO}+[\mathrm{Ni}($ tet $b)]$ along with the estimates of Ni content (from measurements at three different sites for each functionalized material). 

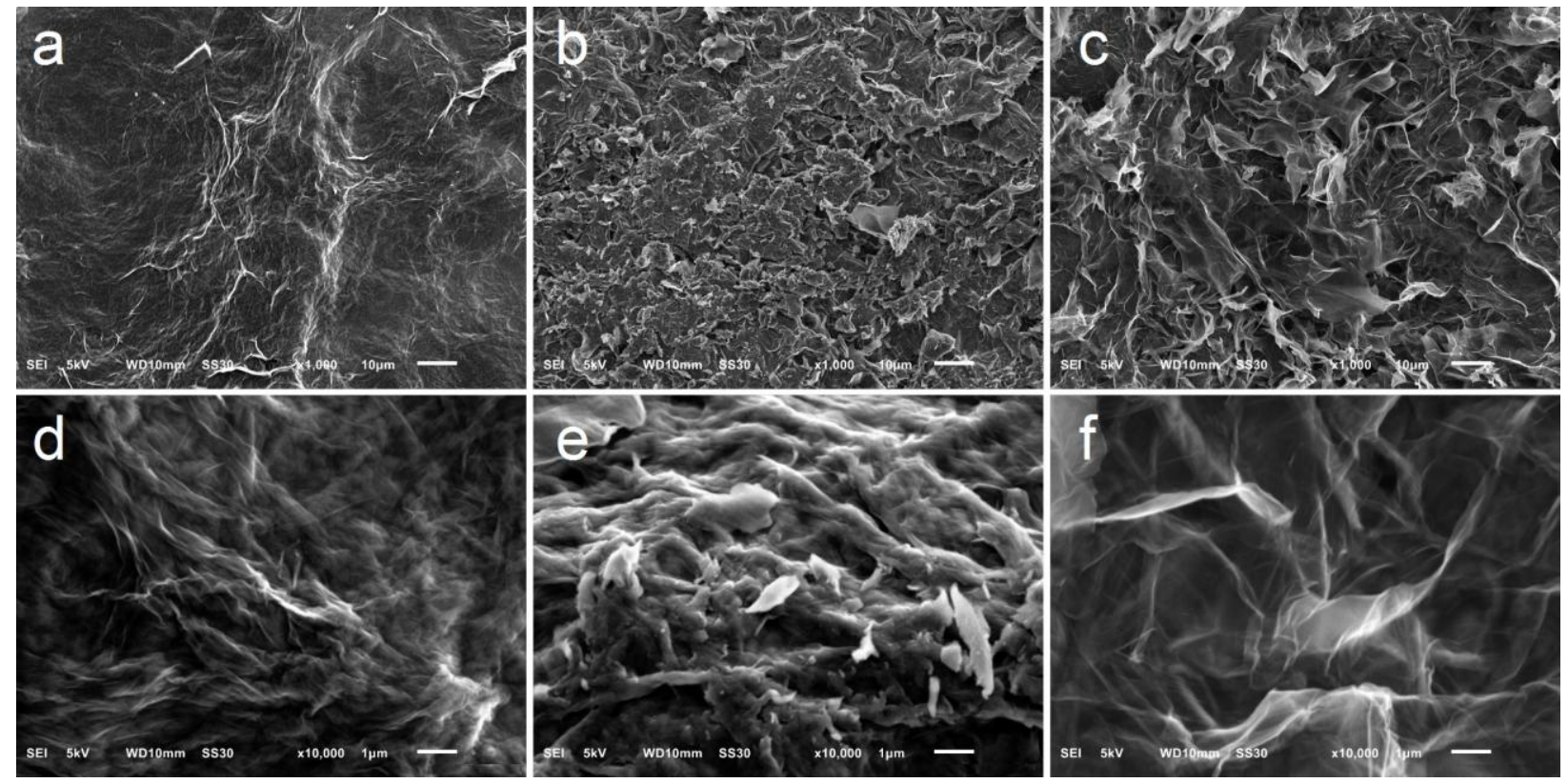

Figure 6. Representative SEM images at different magnifications for (a,d) pristine GO, in comparison with GO samples functionalized with $[\mathrm{Ni}(\text { cyclam })]^{2+}(\mathrm{b}, \mathrm{e})$ and $[\mathrm{Ni}(\text { tet } b)]^{2+}(\mathrm{c}, \mathrm{f})$. Scale bar: (a-c) 10 and (d-f) $1 \mu \mathrm{m}$. 

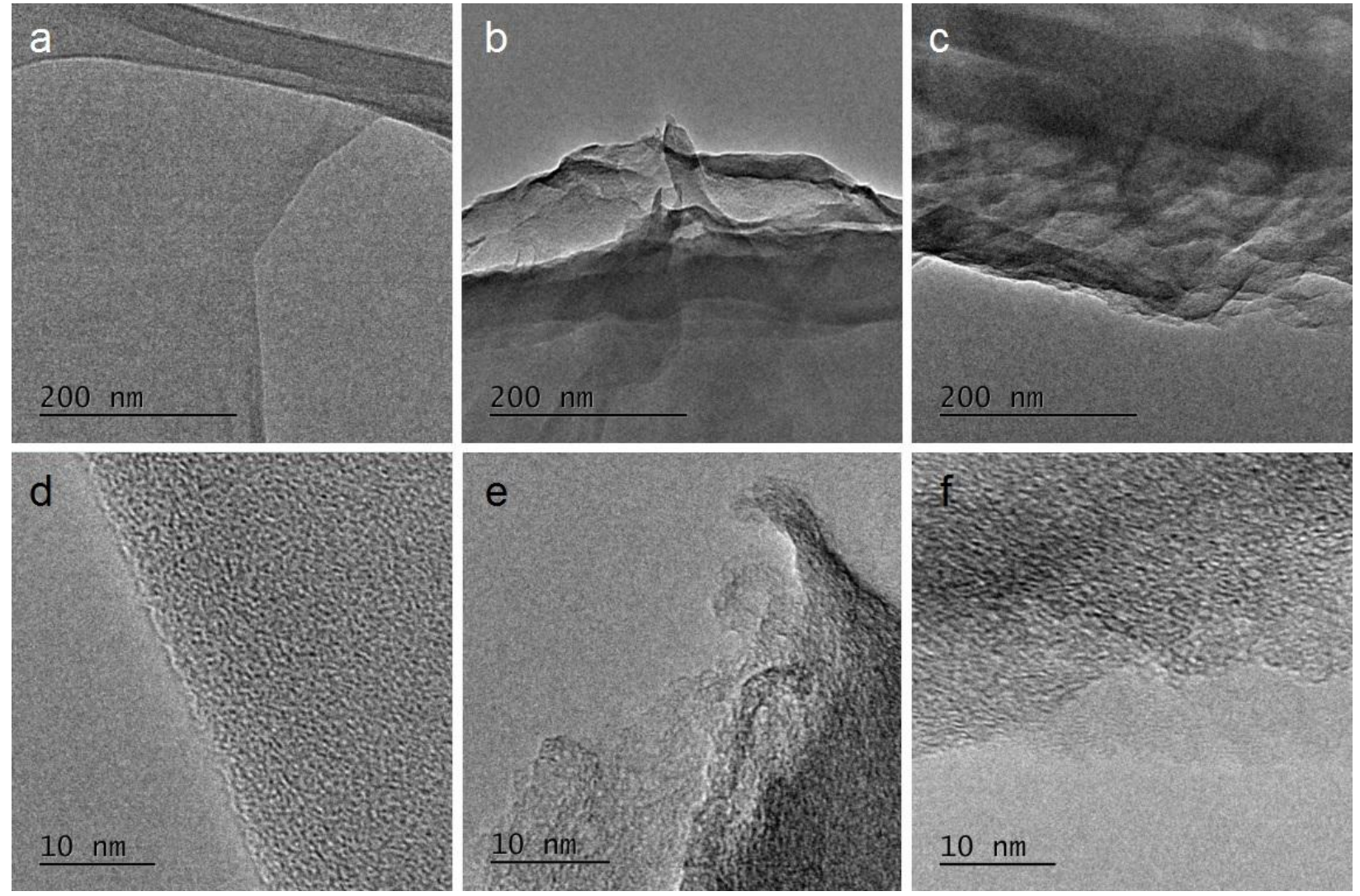

Figure 7. Representative TEM images at different magnifications for (a,d) pristine GO, in comparison with GO samples functionalized with $[\mathrm{Ni}(\text { cyclam })]^{2+}(\mathrm{b}, \mathrm{e})$ and $[\mathrm{Ni}(\text { tet } b)]^{2+}(\mathrm{c}, \mathrm{f})$. 

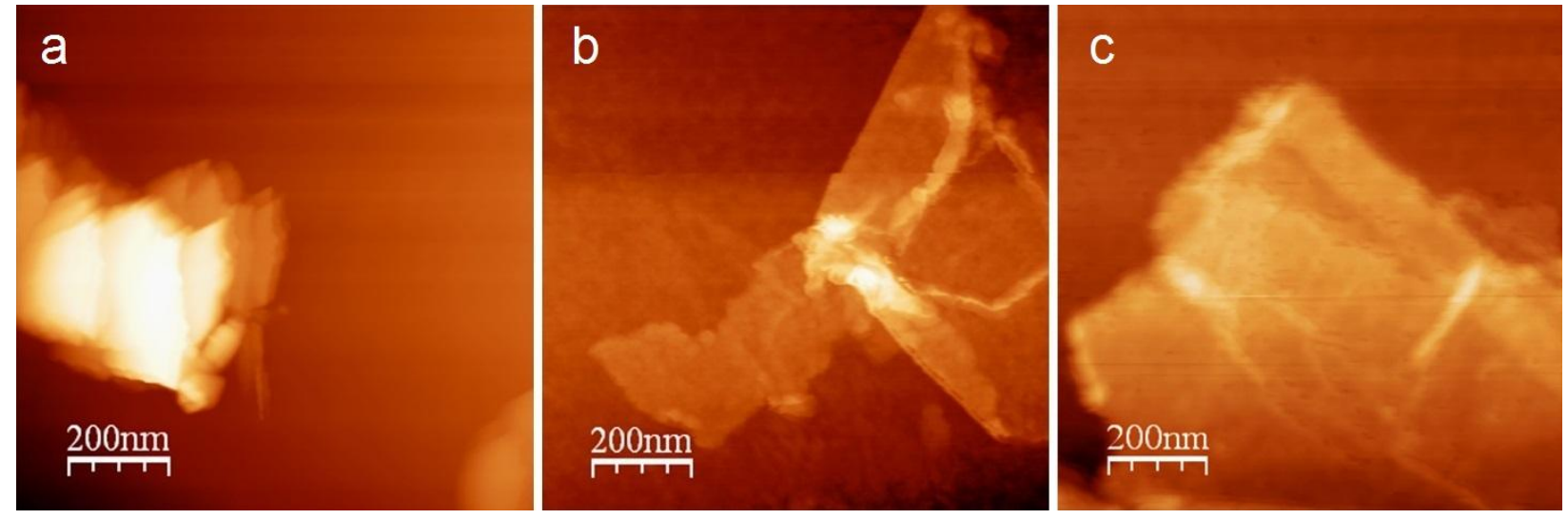

Figure 8. Representative AFM topography images for (a) pristine GO, (b) GO+[Ni(cyclam)] and (c) $\mathrm{GO}+[\mathrm{Ni}($ tet $b)]$. 

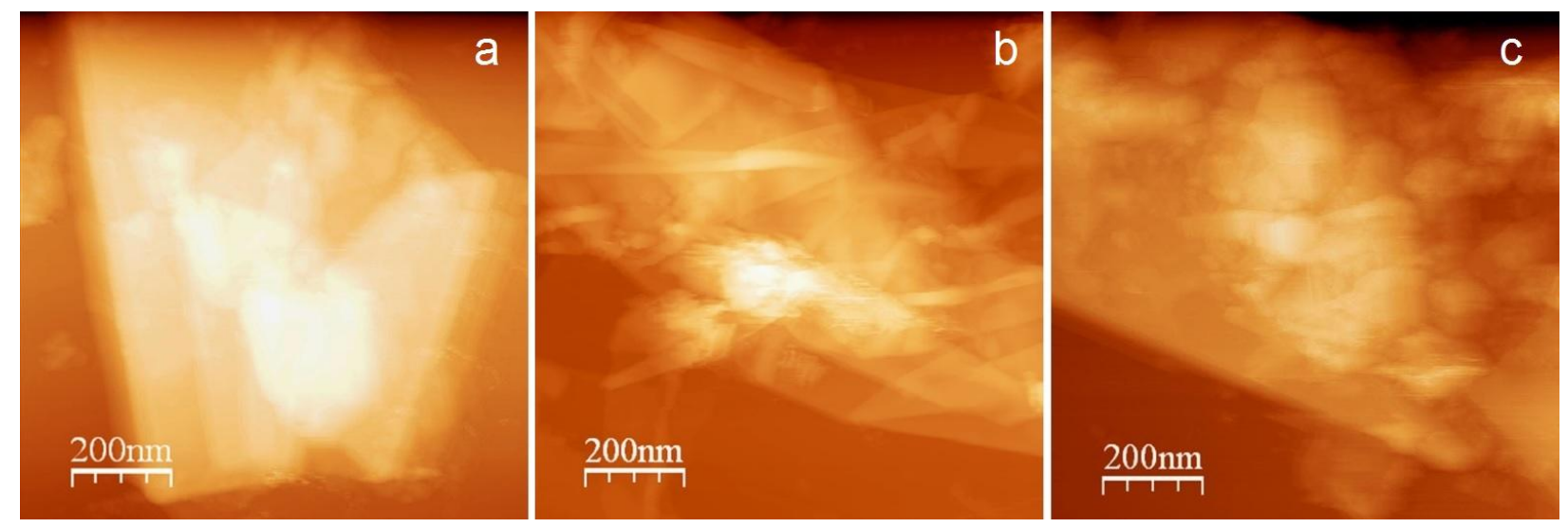

Figure 9. Representative STM images for (a) pristine GO, (b) GO+[Ni(cyclam)] and (c) GO+[Ni(tet b)]. 
GO
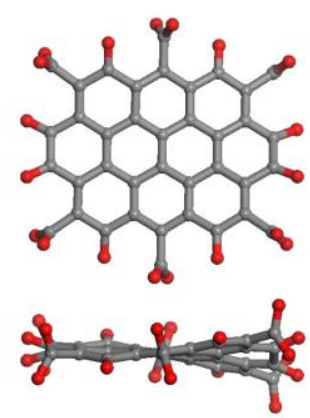

$\mathrm{HOMO}$

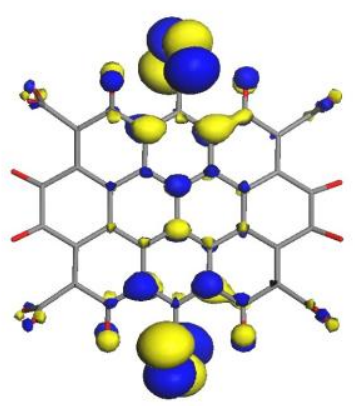

LUMO

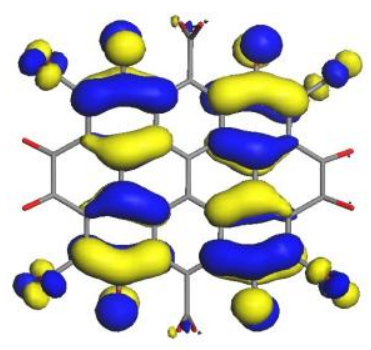

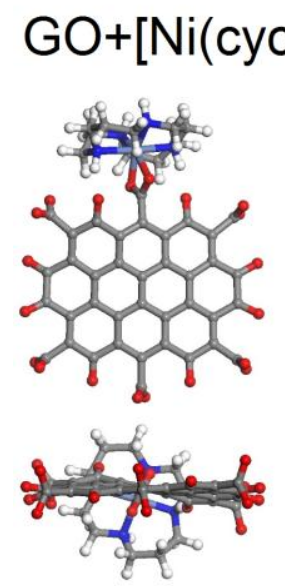

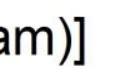

HOMO

LUMO

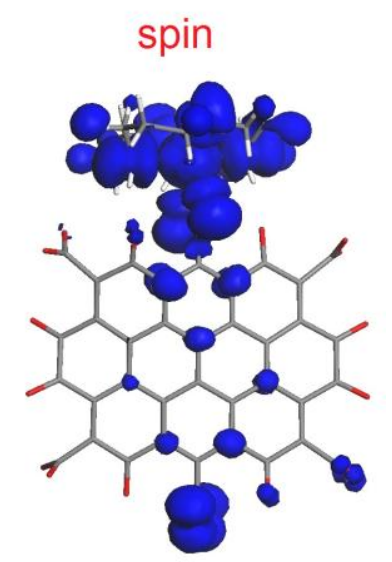

$\mathrm{GO}+[\mathrm{Ni}($ tet $b)]$
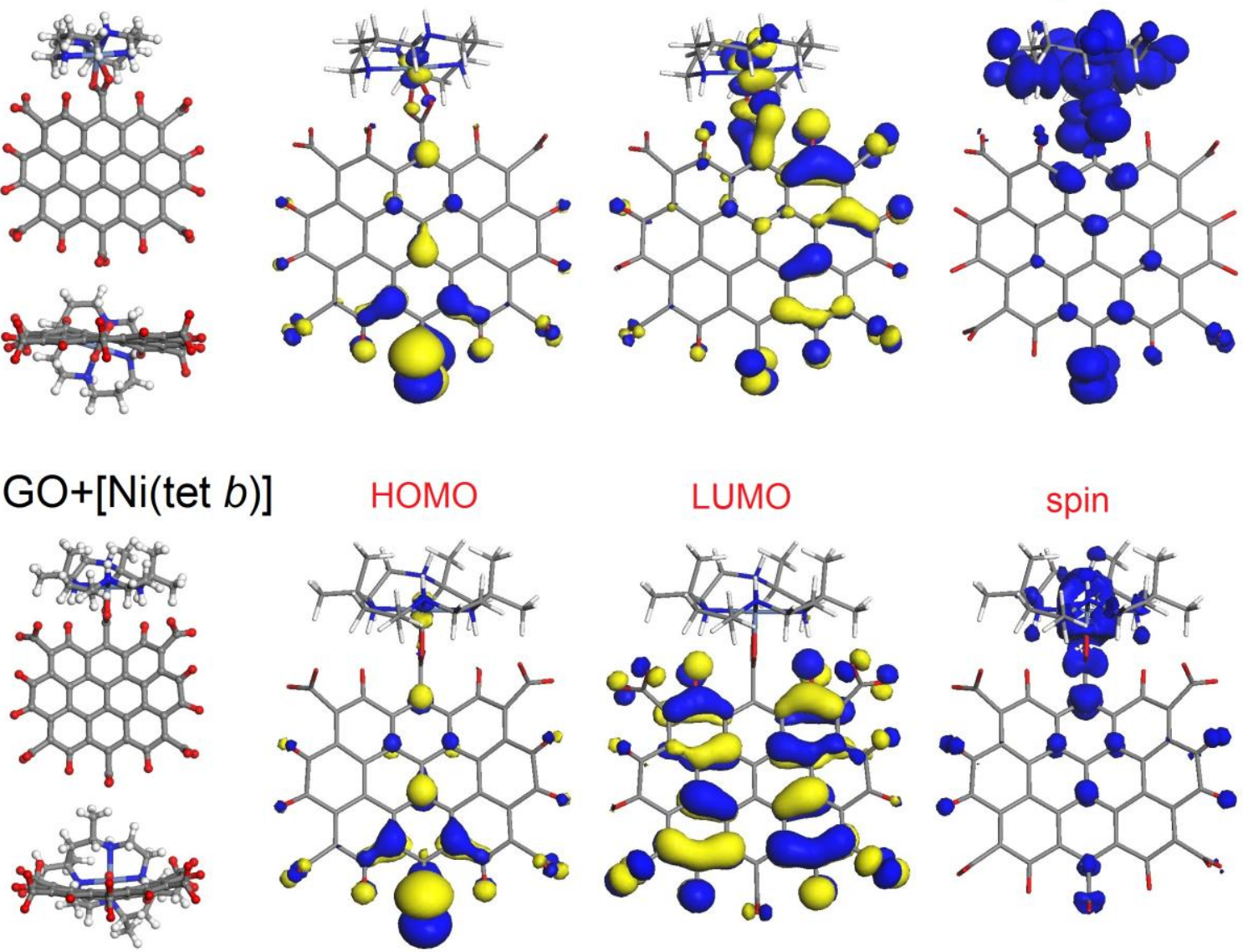

Figure 10. Optimized structures (top and edge views on the left) of the models for GO sheet, $\mathrm{GO}+[\mathrm{Ni}(\mathrm{cyclam})]$ and $\mathrm{GO}+[\mathrm{Ni}($ tet $b)]$ complexes, along with the respective calculated HOMO and LUMO plots (isosurfaces at 0.02 a.u.) and spin density distribution (isosurfaces at 0.001 a.u.) for the complexes. All the carboxylic groups are deprotonated, as in a basic medium, resulting in the total charge of 6- for GO model, and 4- for the complexes GO+[Ni(cyclam)] and GO+[Ni(tet $b$ )]. 